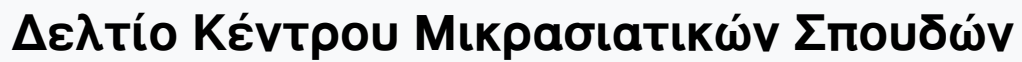

Tóp. 1 (1977)

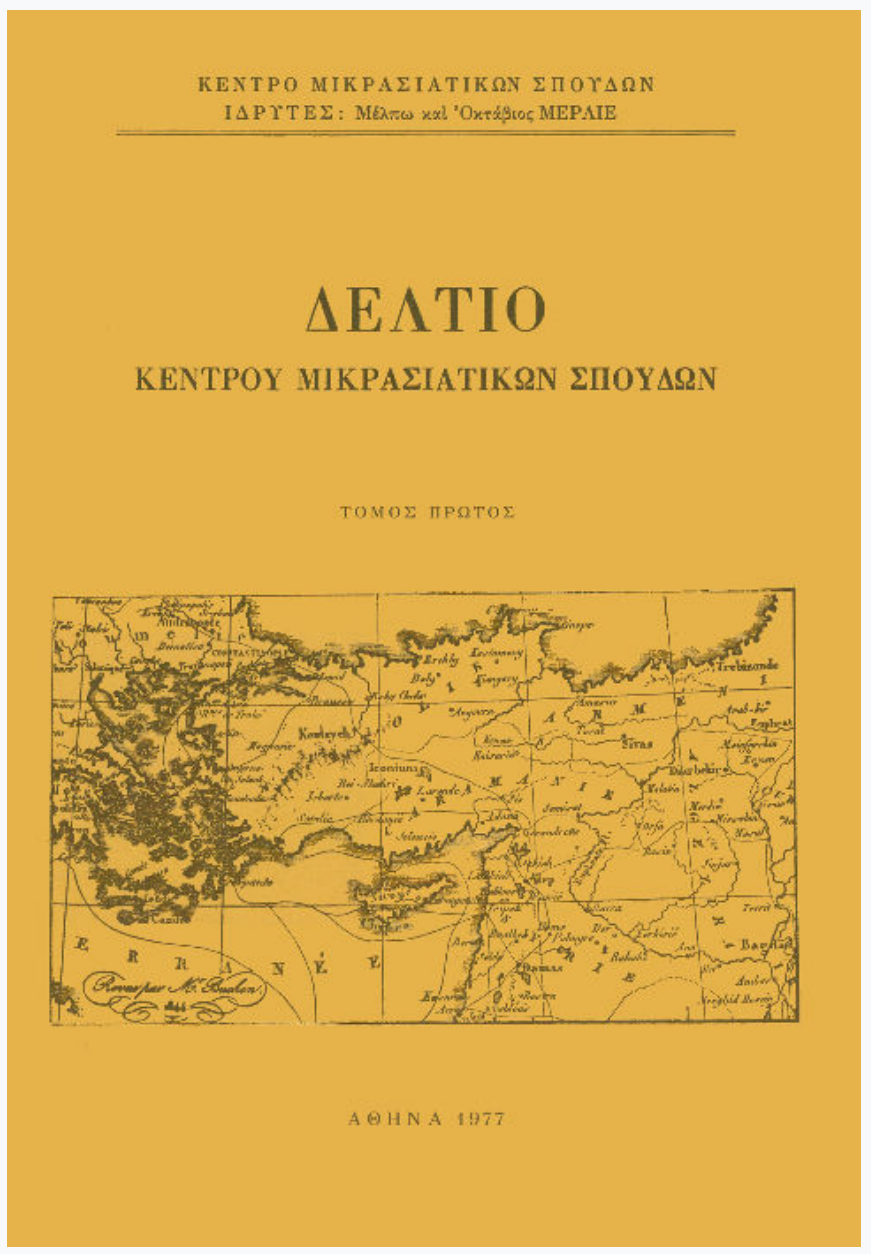

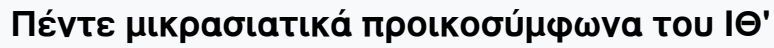
aı́́va

\section{Mapía laкẃßou-Oıкоvómou}

doi: $10.12681 /$ deltiokms.175

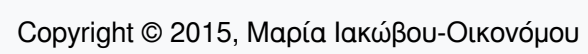

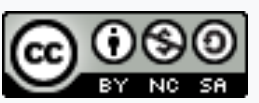

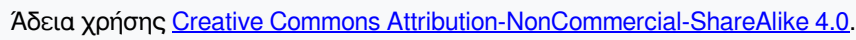

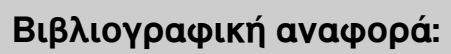

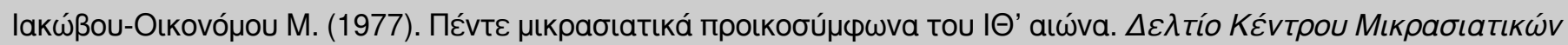

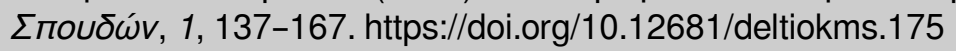




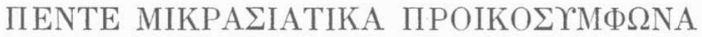

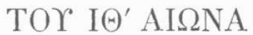

\author{
$\tilde{r}_{\zeta}$ Map. IAK $\Omega B O r-O I K O N O M O r$
}

\section{$\mathrm{A}^{\prime} . \mathrm{EI} \Sigma \mathrm{A} \Gamma \Omega \Gamma \mathrm{H}$}

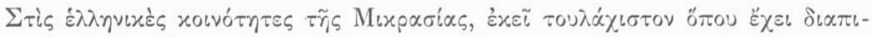

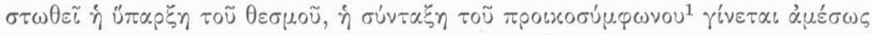

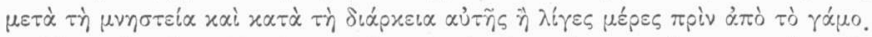

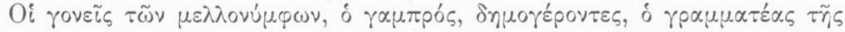

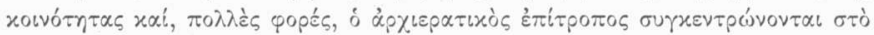

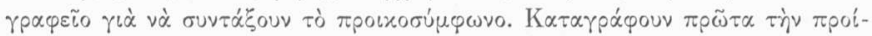

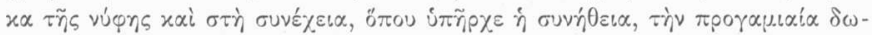

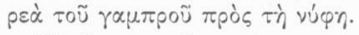

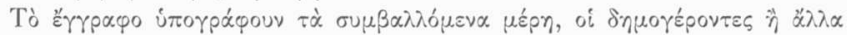

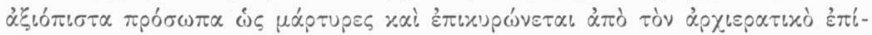

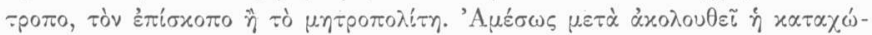

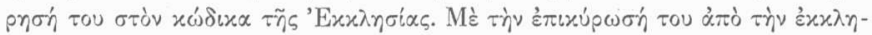

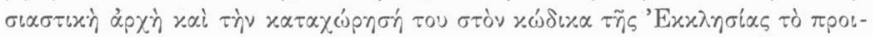

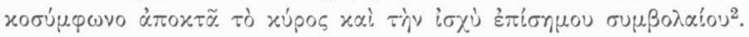

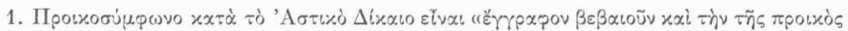

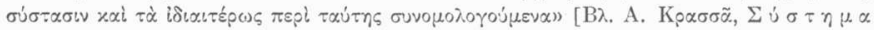

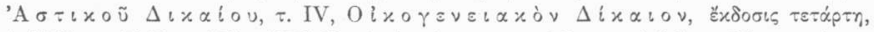
¿̇v 'A0ท்

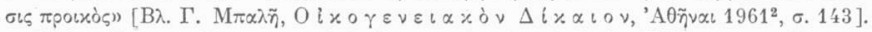

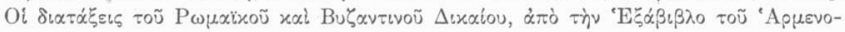

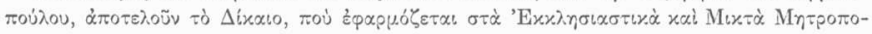

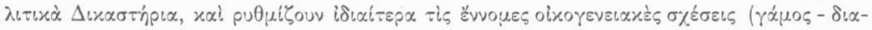

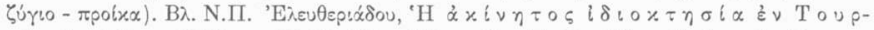
$x\lfloor\alpha$, ¿̇v 'A

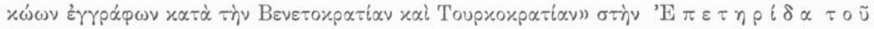

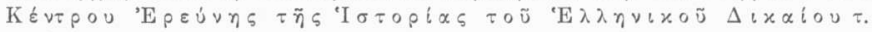

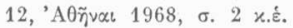

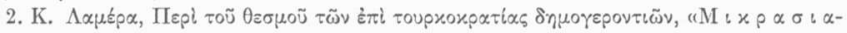




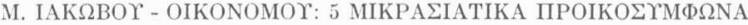

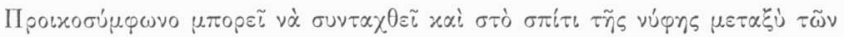

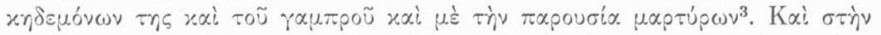

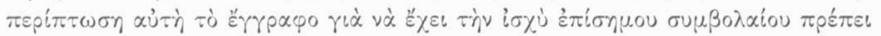

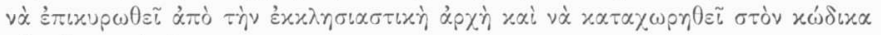

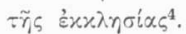

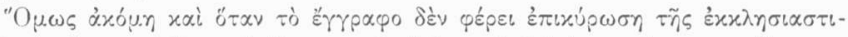

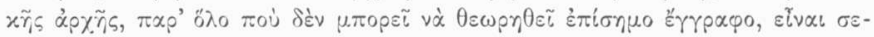

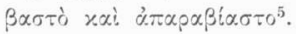

$$
* * *
$$

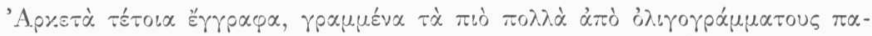

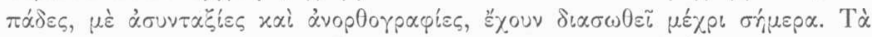

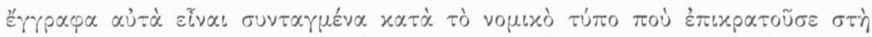

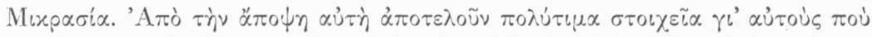

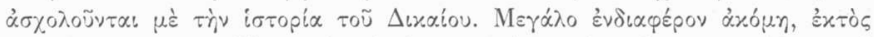

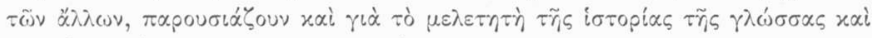

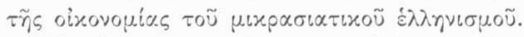

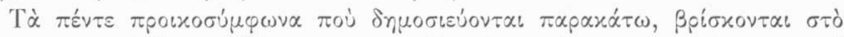

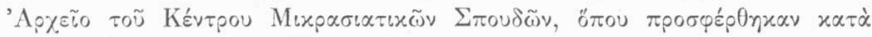

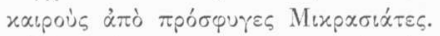

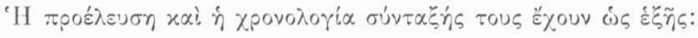

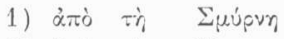
9 'Iovíou 1841
2) $\alpha \pi \dot{~}$ тò M $\alpha \rho \mu \alpha \rho \tilde{\alpha}$

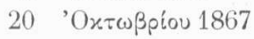
3) $\alpha \pi \dot{c} \tau i \dot{v}$ 'Aprćxy
19 'Iavouxpiou 1874

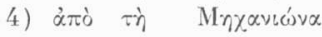
11 'A
5) $\alpha \pi \dot{~ \tau i ̀ v ~ ' A q u \sigma ı ̀ ~}$
18 Фвßроu opíou 1899

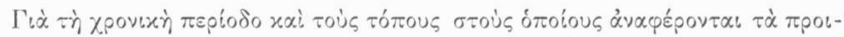

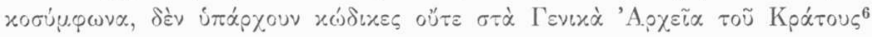

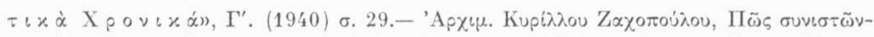

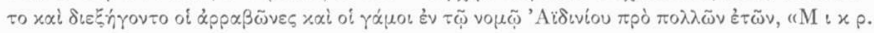

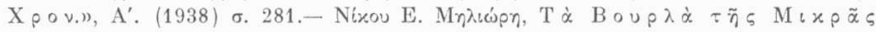

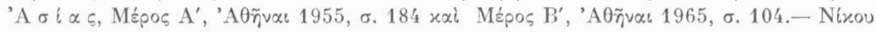

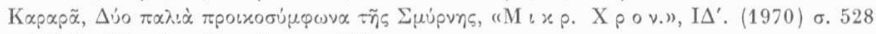

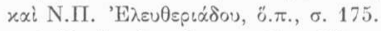

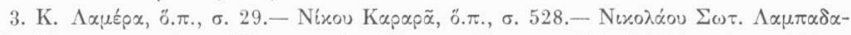

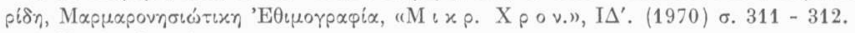

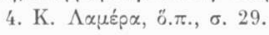

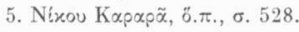

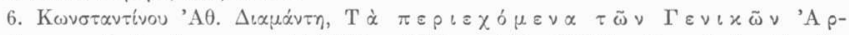

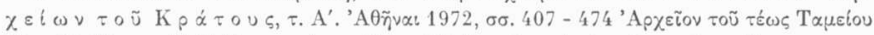

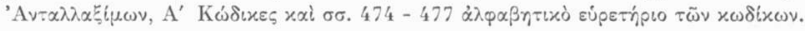




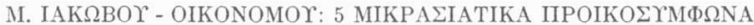

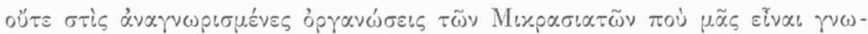

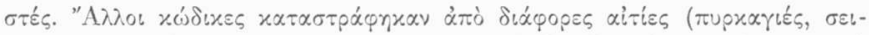

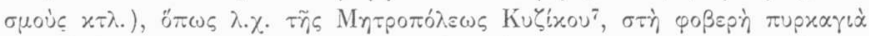

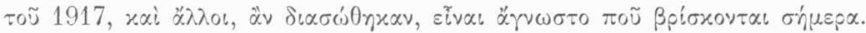

$$
\text { *** } *
$$

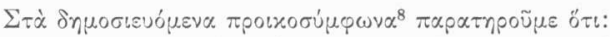

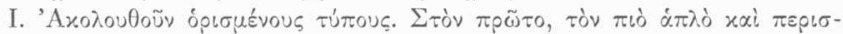

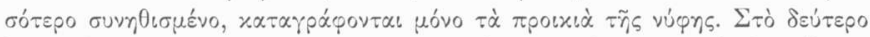

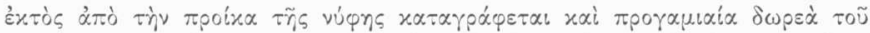

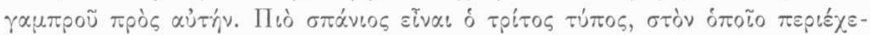

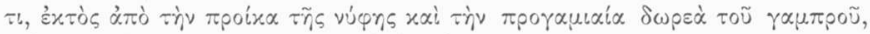

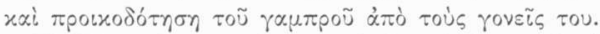

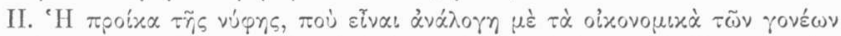

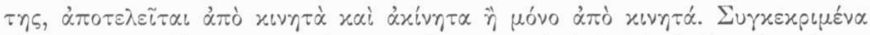

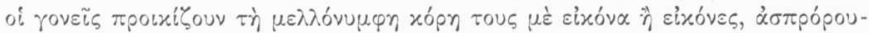

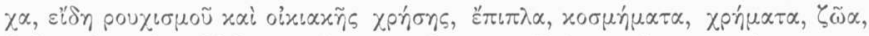

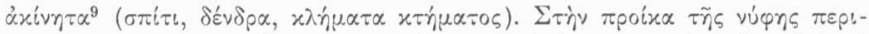

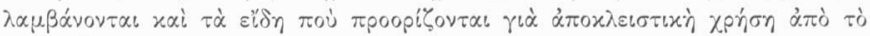
$\gamma \propto \mu \pi \rho \dot{0}$.

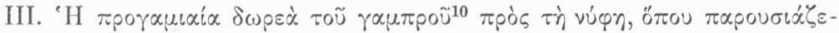

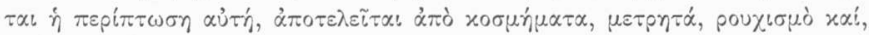
$\sigma \pi \dot{\alpha} v i \alpha, \alpha \dot{x} i v \eta \tau \alpha$.

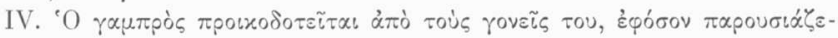

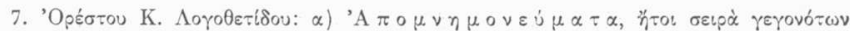

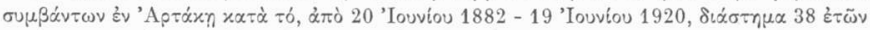

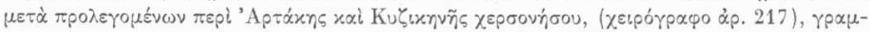

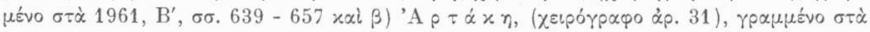
1954, б. 48 - 49.- К

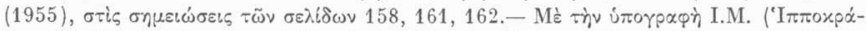

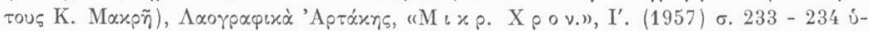

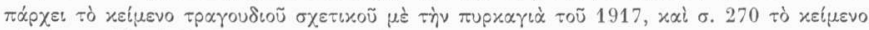

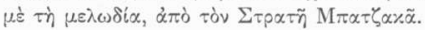

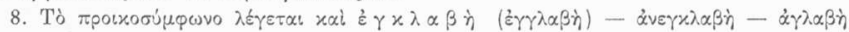

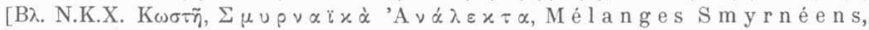

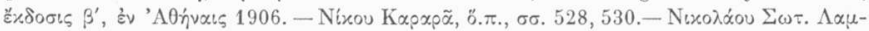

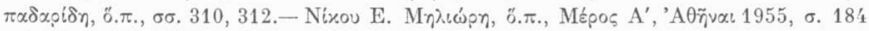
xхi Mépos B', 'A

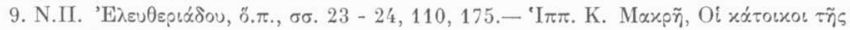

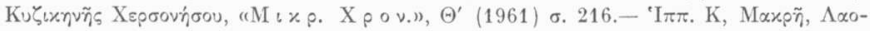

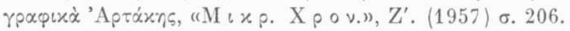

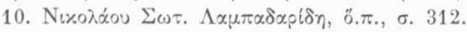




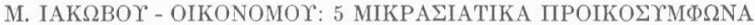

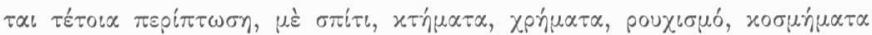

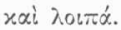

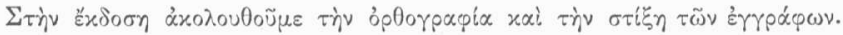

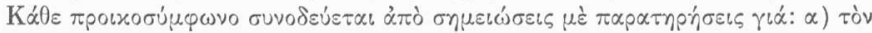

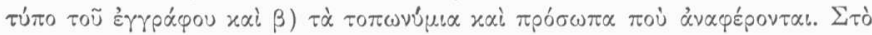

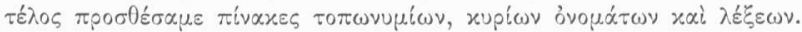

\section{B'. TA KEIMENA}

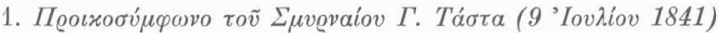

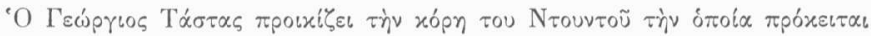

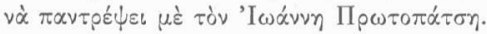

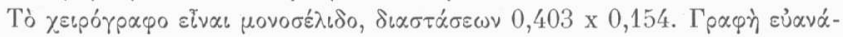
$\gamma \vee \omega \sigma \tau \eta$.

\section{ВIBАIОГРАФIA}

'E $\varphi \eta \mu$. "M

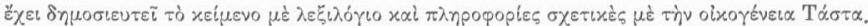

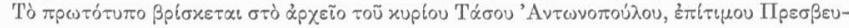
$\tau \tilde{\eta}$.

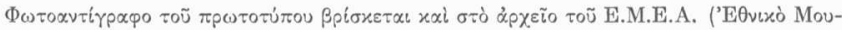

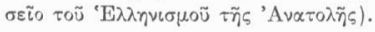

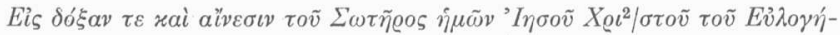

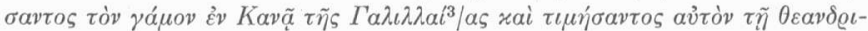

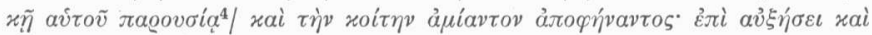

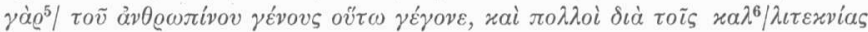

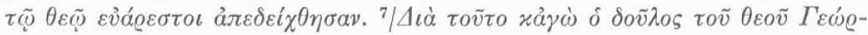

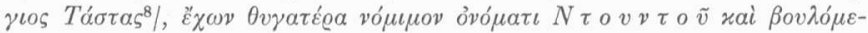

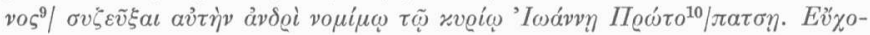

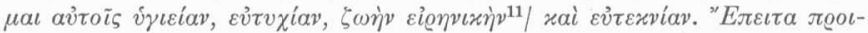

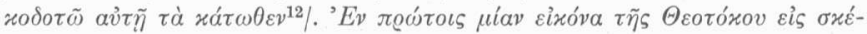

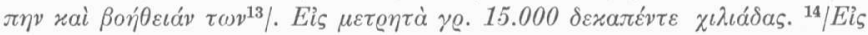

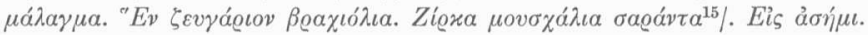

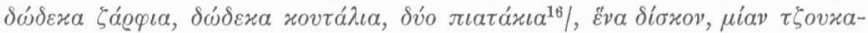

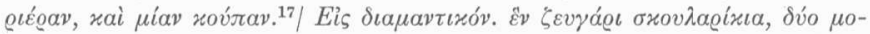

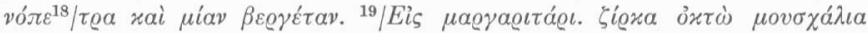

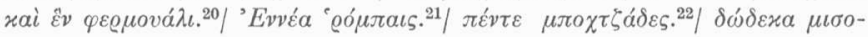




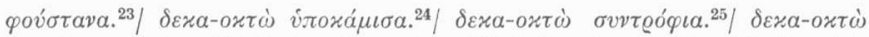

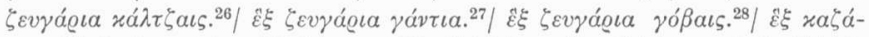

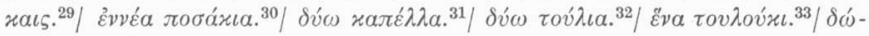

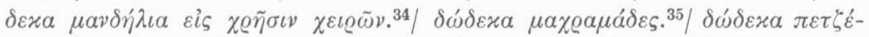

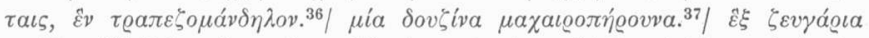

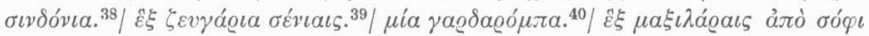

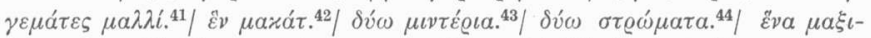

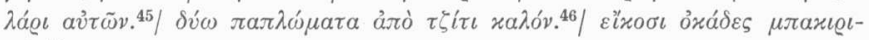
róv. ${ }^{74}$

\section{$\Sigma \mu v ́ \varrho v \eta$ т̀े 9 'Iovגiov 1841}

$\Gamma \varepsilon \omega . \Sigma \alpha \dot{\beta} \beta \alpha \varsigma \mu \alpha \varrho \tau v \varrho \tilde{\omega}$

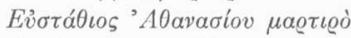

$\Delta \eta \mu \dot{r} \tau \varrho \iota s, \mu \iota \chi \alpha \dot{\lambda} \lambda \mu \alpha \varrho \tau \iota$.

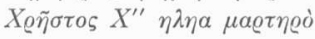

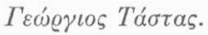

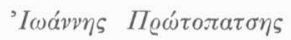

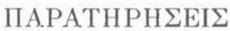

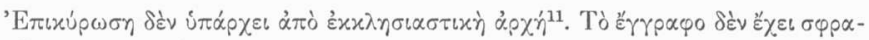

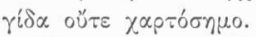

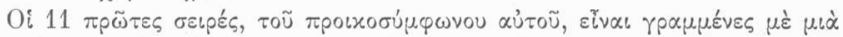

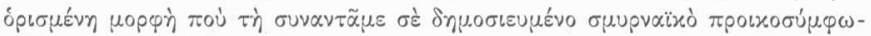

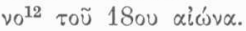

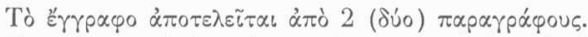

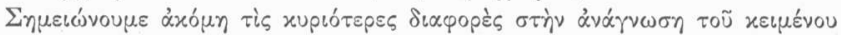

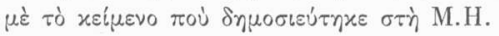

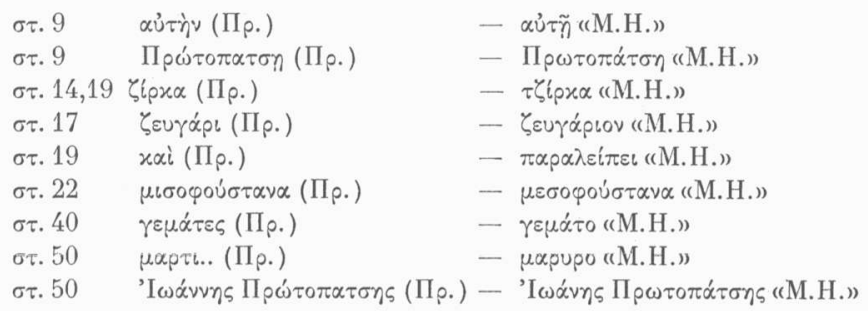

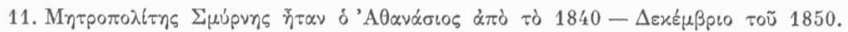

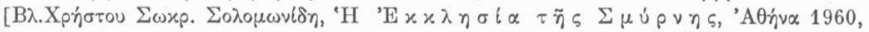

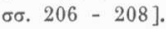

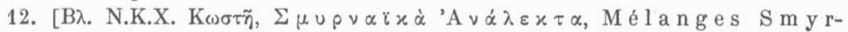

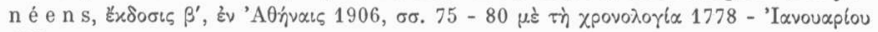
$18]$. 


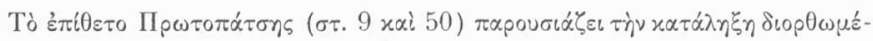

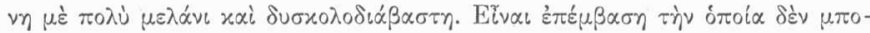

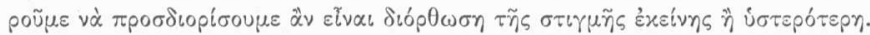

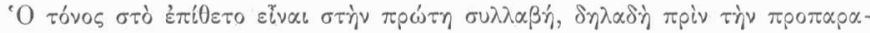
$\lambda \dot{\gamma} \gamma \sigma u \sigma \alpha \quad(\Pi \rho \dot{\omega} \tau 0 \pi \alpha \tau \sigma \eta \xi)$.

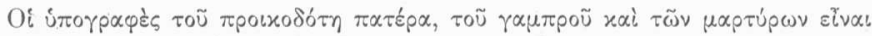

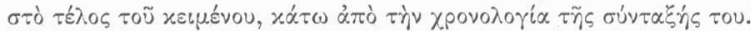

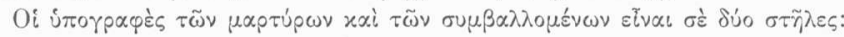

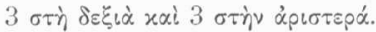

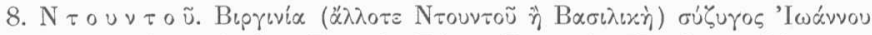

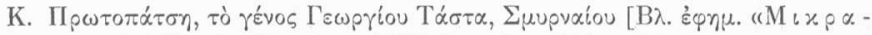

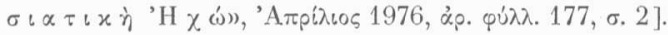

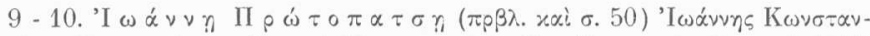

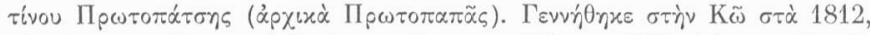

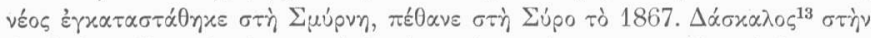

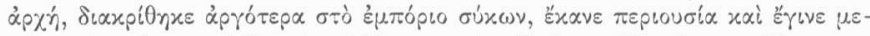

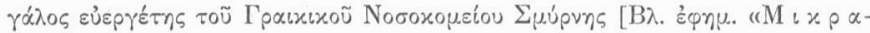

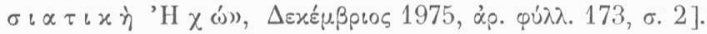

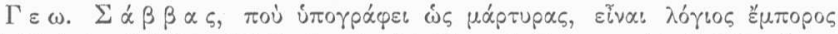

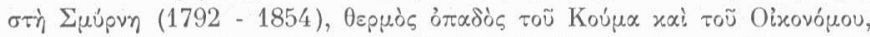

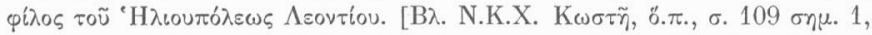

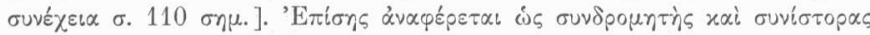

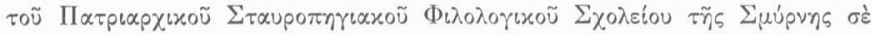

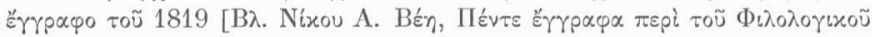

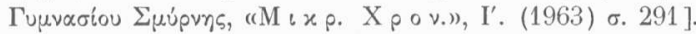

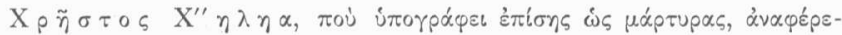

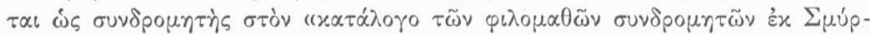

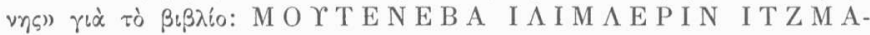

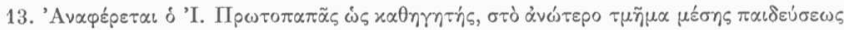

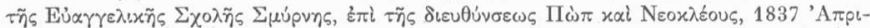

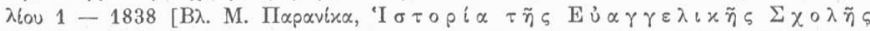

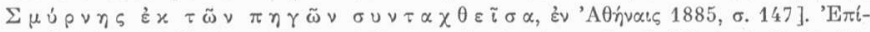

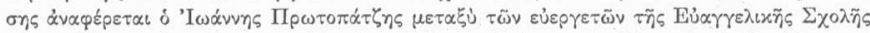

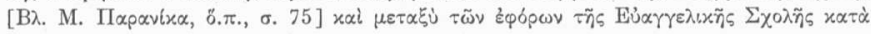

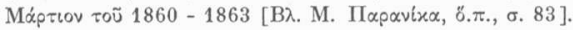




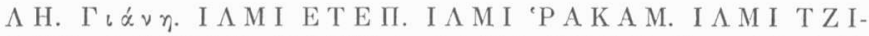

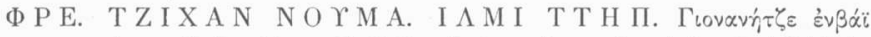

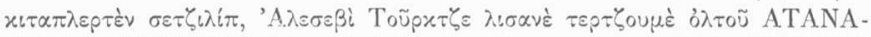

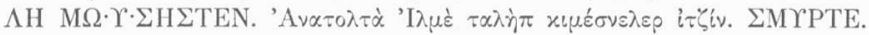

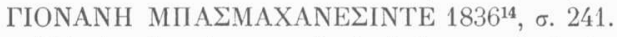

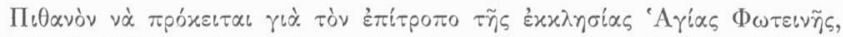

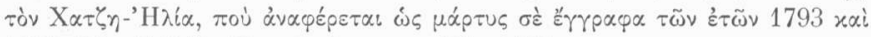

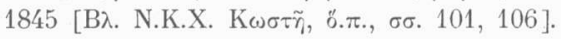

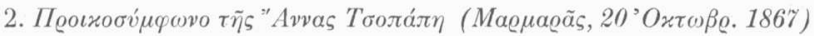

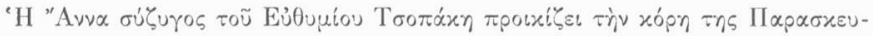

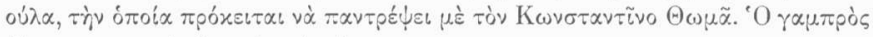

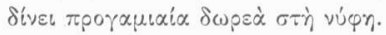

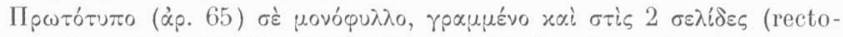

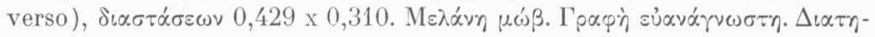

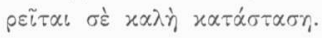

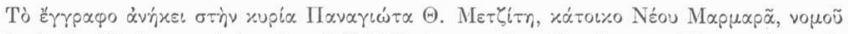

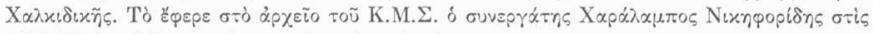

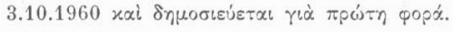

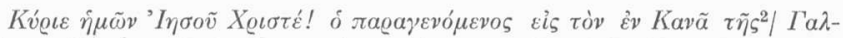

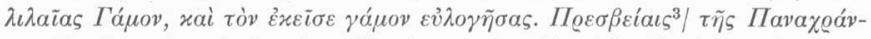

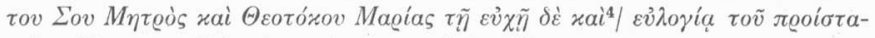

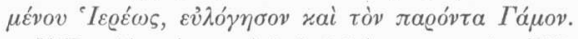

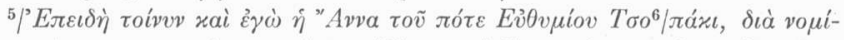

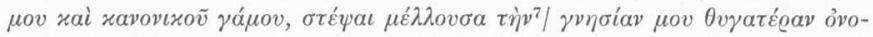

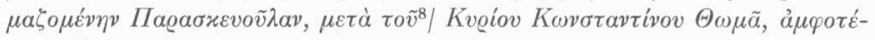

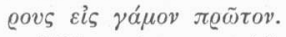

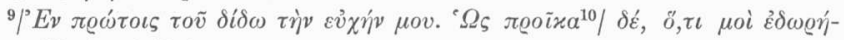
бато Kvoíos o @eós.

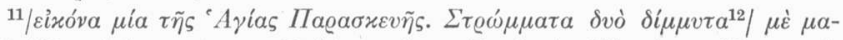

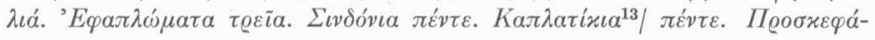

14. $\Sigma \pi \circ \cup \delta \alpha i \alpha \Sigma \dot{u} v \sigma \psi \eta \quad \mathrm{E} \pi \iota \sigma \tau \eta \mu \tilde{\omega} v, \delta \eta \lambda \alpha \delta \dot{\eta}$ 'H $\theta \iota x \dot{\varepsilon} \varsigma$ ' $\mathrm{E} \pi \iota \sigma \tau \tilde{\eta}-$

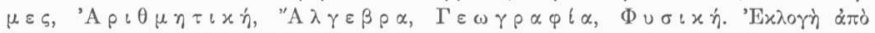

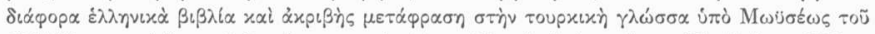

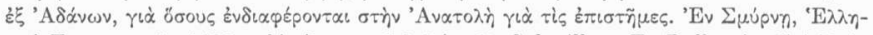

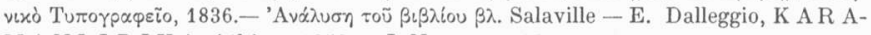

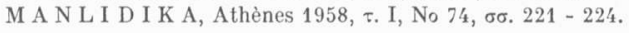




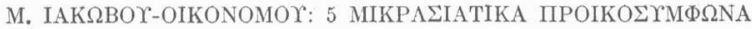

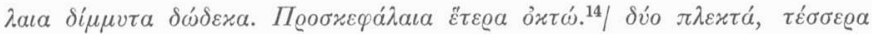

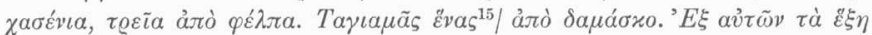

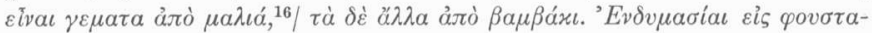

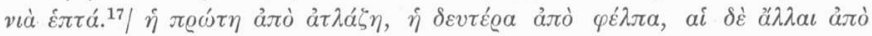

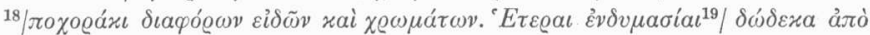

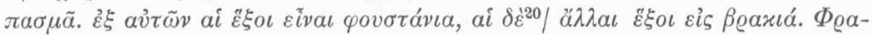

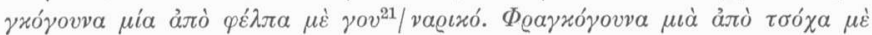

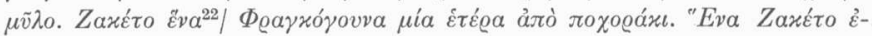

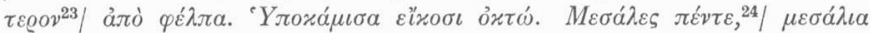

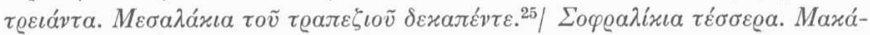

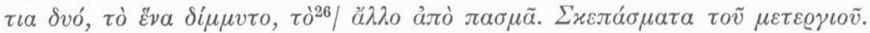

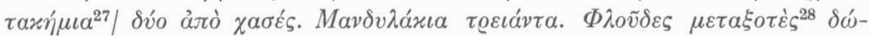

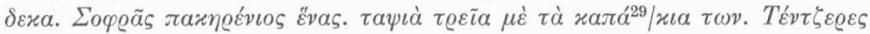

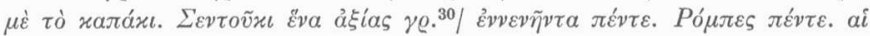

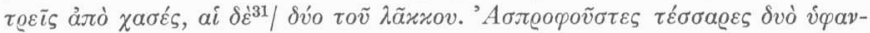

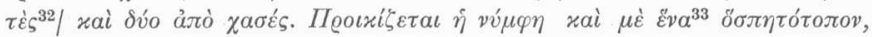

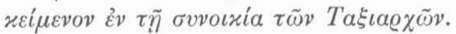

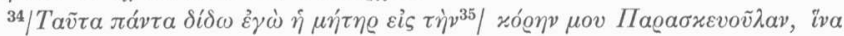

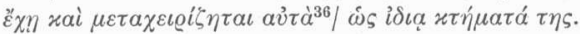

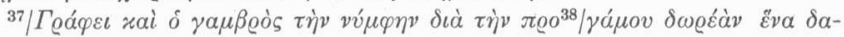

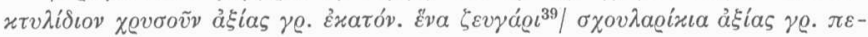

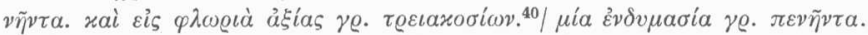

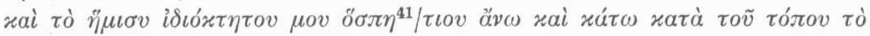

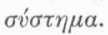

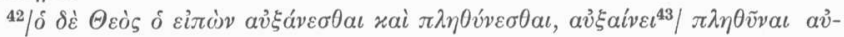

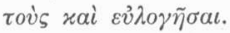

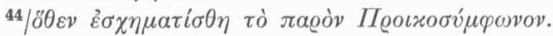

Oi $\pi \alpha \varrho \varepsilon v \varrho \varepsilon \theta \varepsilon \dot{v} \tau \varepsilon \varsigma$

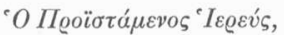

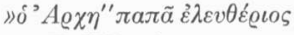

" $\Pi a^{\prime \prime}$ Kveıáxos

$\dot{\eta} \Delta \eta \mu о \gamma \varepsilon \varrho o \nu \tau i a$

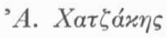

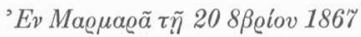

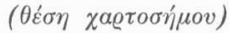

$\tau \dot{\alpha} \sigma v \mu \beta \alpha \lambda \lambda o \mu \varepsilon v \dot{\alpha} \mu \varepsilon \varrho \dot{\eta}$

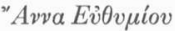

$K \omega \sigma \tau \alpha \tau \tilde{\eta} \varsigma \Theta \omega \mu \tilde{\alpha}$

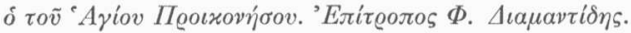




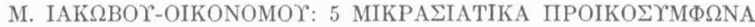

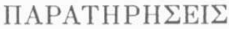

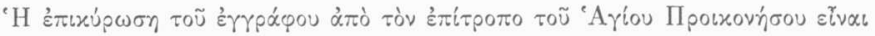

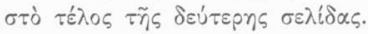

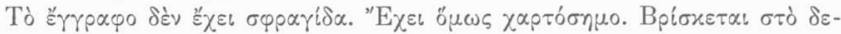

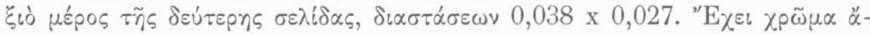

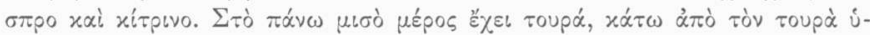

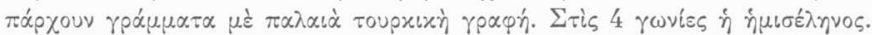

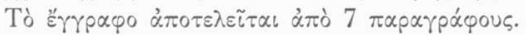

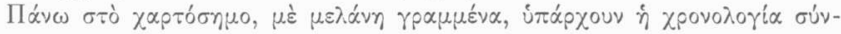

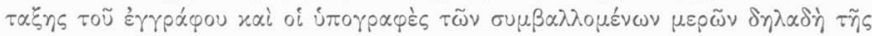

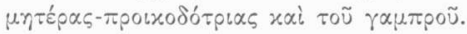

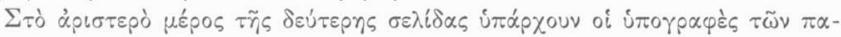

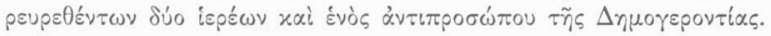

33. $\sigma \cup \vee \circ \iota x i \alpha \tau \tilde{\omega} \nu \mathrm{T} \alpha \xi \iota \alpha \rho \chi \tilde{\omega} v$. 'H

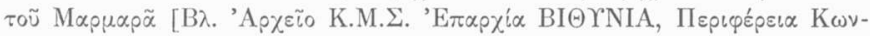

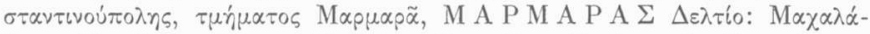

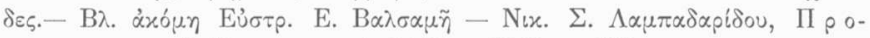

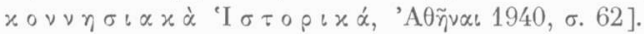

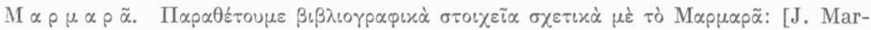

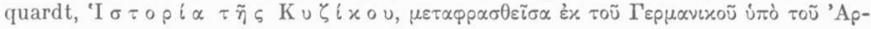

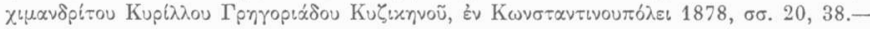

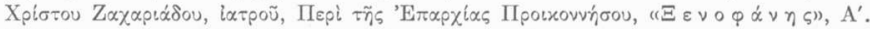

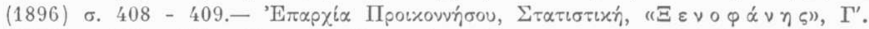

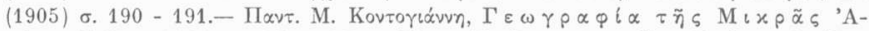

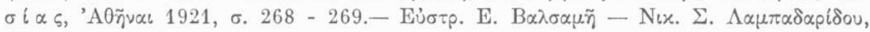

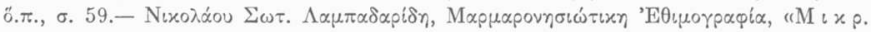
$\mathrm{X} \rho \circ$ v.1), I $\left.\Delta^{\prime} .(1970) \sigma \sigma .273-275\right]$.

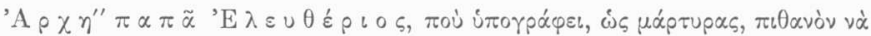

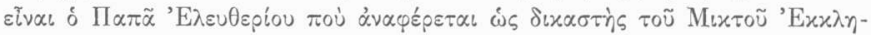

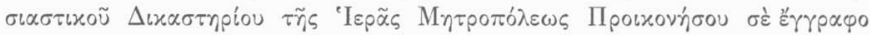

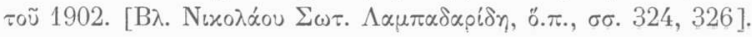

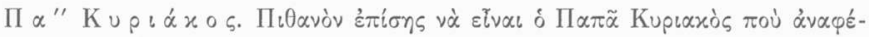

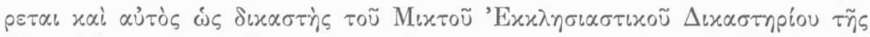

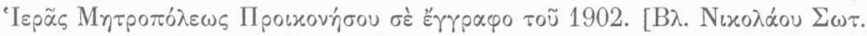

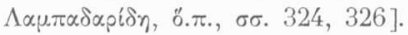

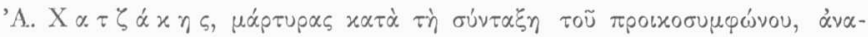

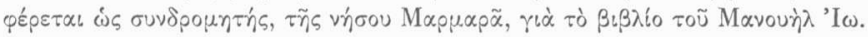




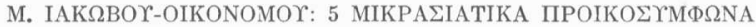

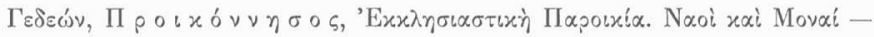

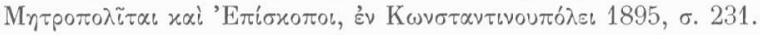

"A Ү

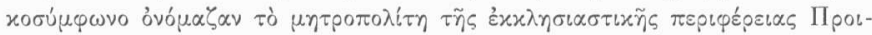

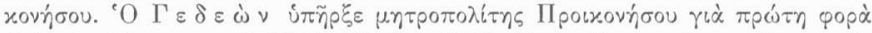

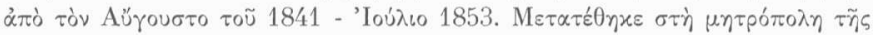

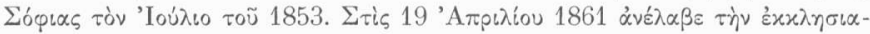

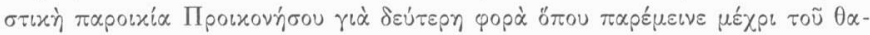

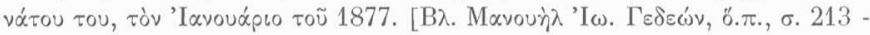
$214]$.

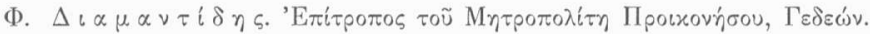

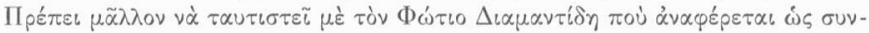

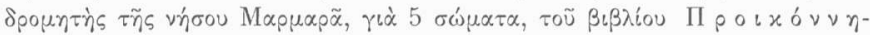

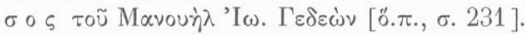

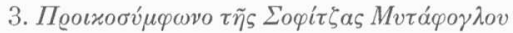

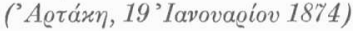

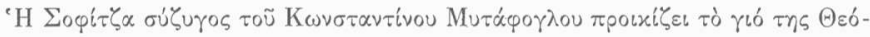

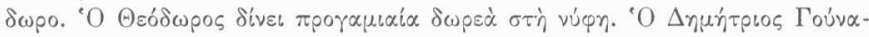

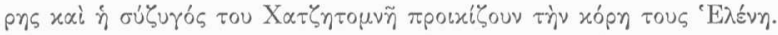

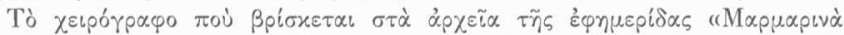

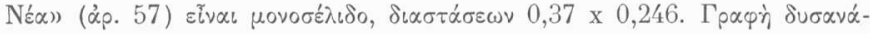

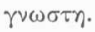

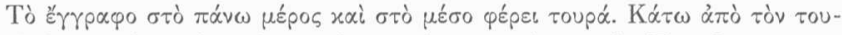

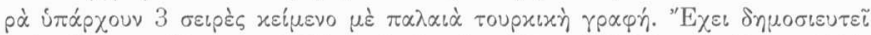

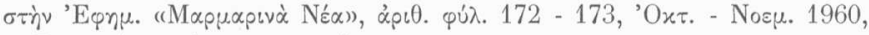

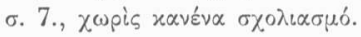

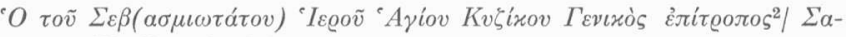

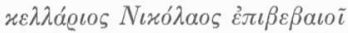

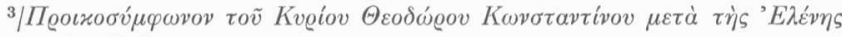
$\triangle \eta \mu \eta \tau \varrho i o v-\Gamma o u ́ v a \varrho \eta$

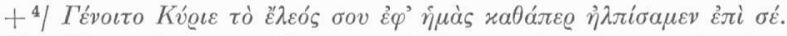

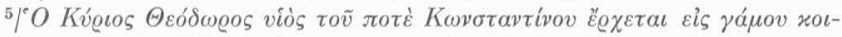

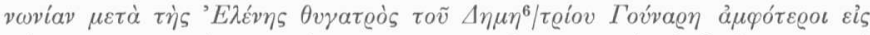

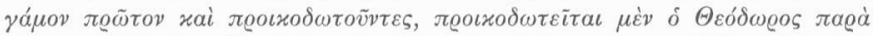

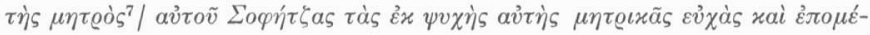




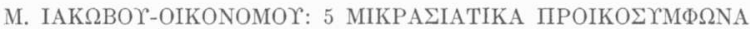

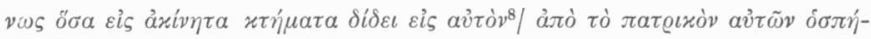

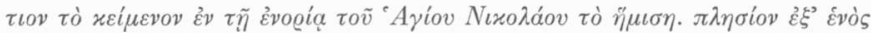

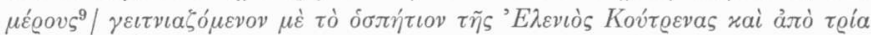

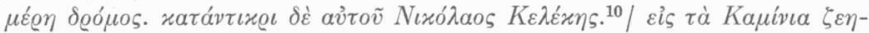

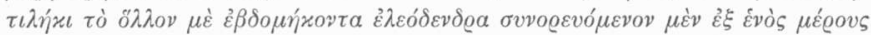

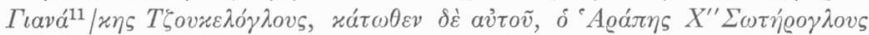

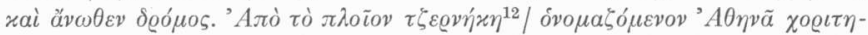

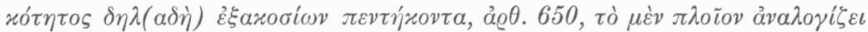

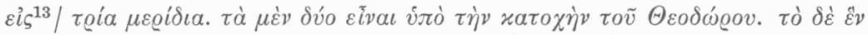

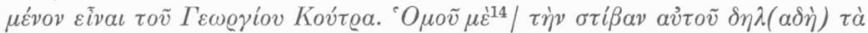

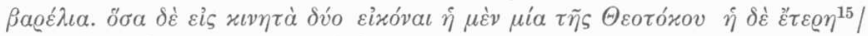

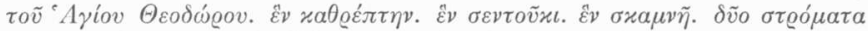

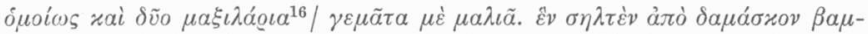

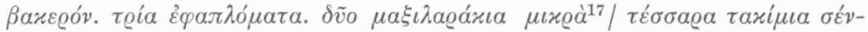

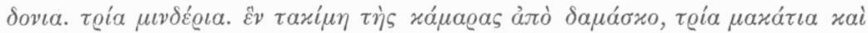

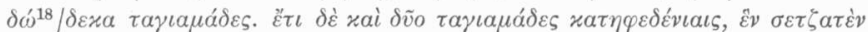

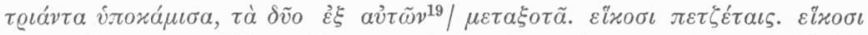

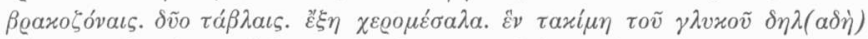

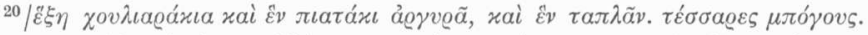

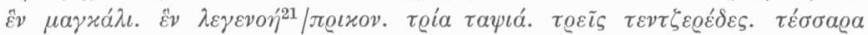

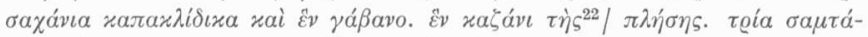

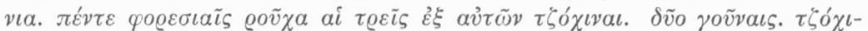

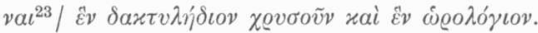

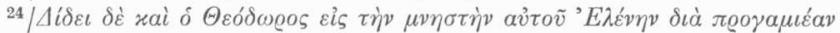

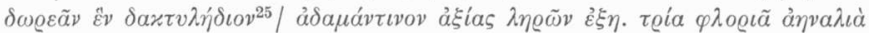

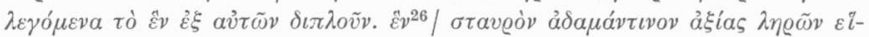

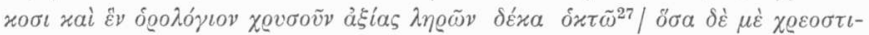

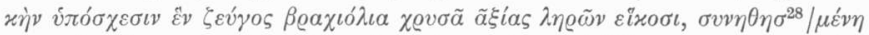

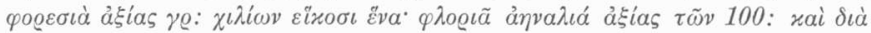

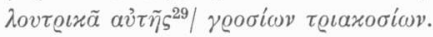

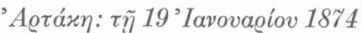

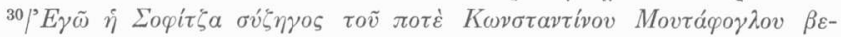

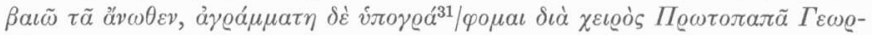
jíov.

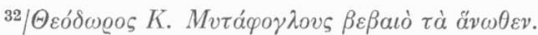

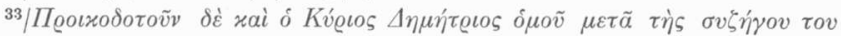

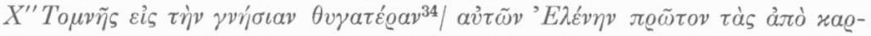

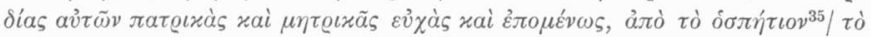

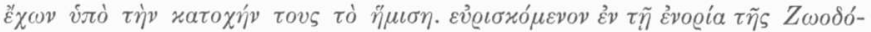

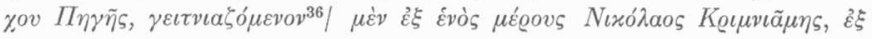




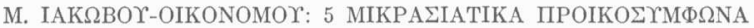

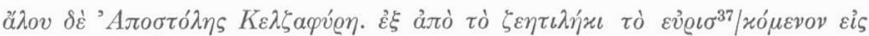

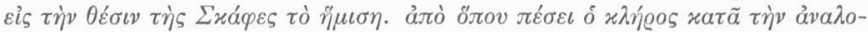

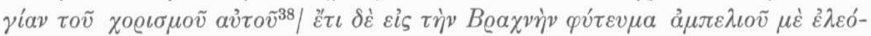

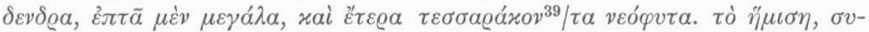

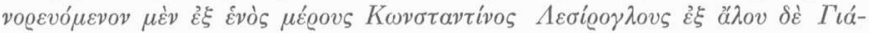

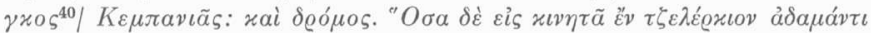

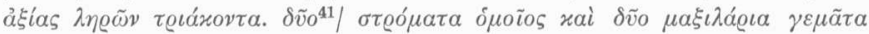

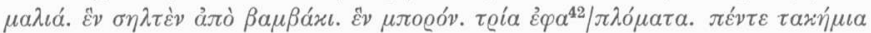

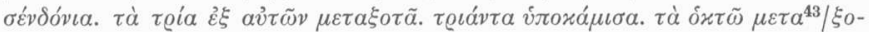

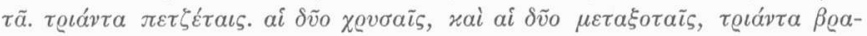

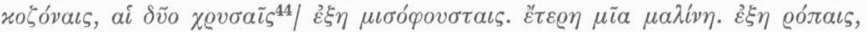

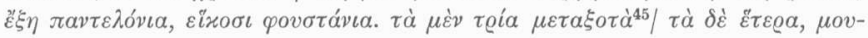

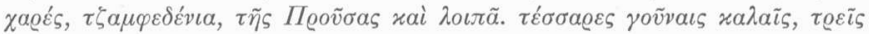

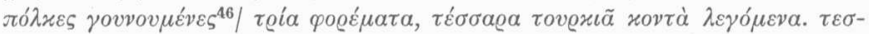

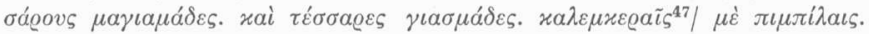

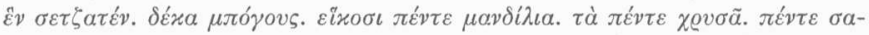

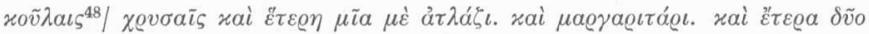

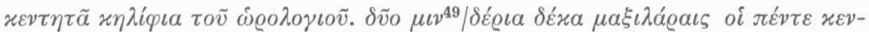

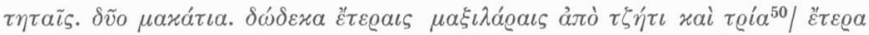

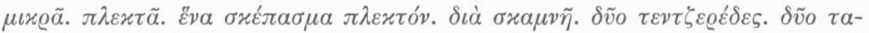

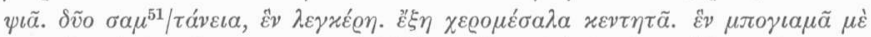

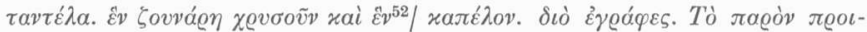

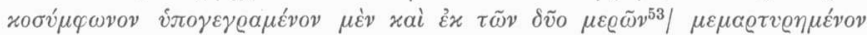

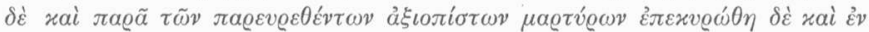

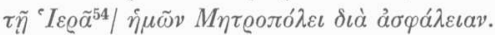

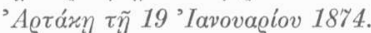

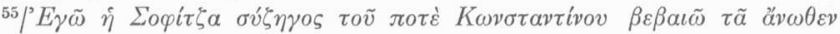

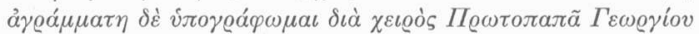

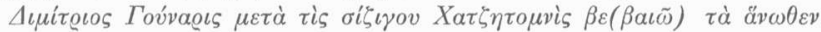

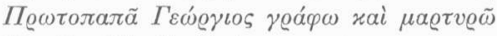

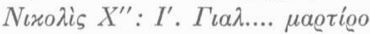

\section{ПАPATHPH $\Sigma$ EI $\Sigma$}

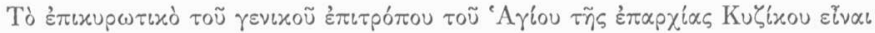

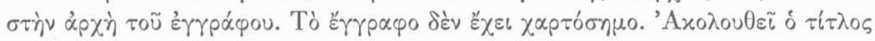
$\mu \dot{\varepsilon} \tau \dot{\alpha}$ óvó $\mu \alpha \tau \alpha \tau \tilde{\omega} \nu \mu \varepsilon \lambda \lambda \alpha^{2} u \dot{\mu} \mu \omega \omega$.

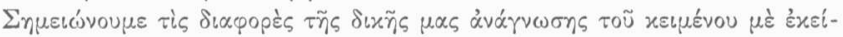

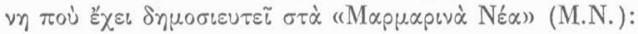




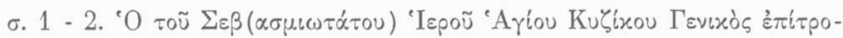

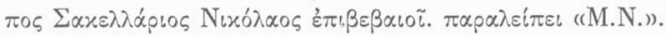

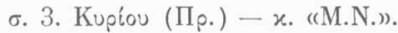

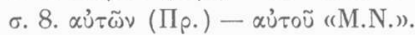

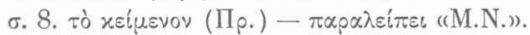

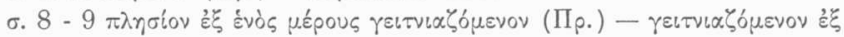

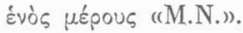

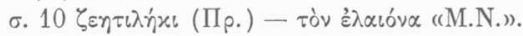

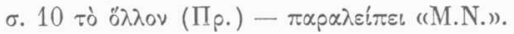

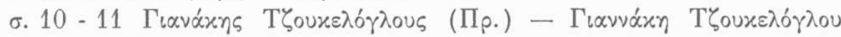
(M.N.).

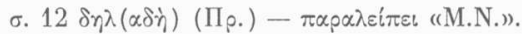

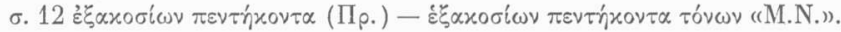

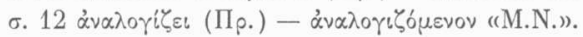

б. $14 \delta \eta \lambda(\alpha \delta \dot{\eta})(\Pi \rho)-.(;) \pi \alpha p \alpha \lambda \varepsilon i \pi \varepsilon \iota$ (M.N.».

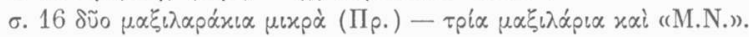

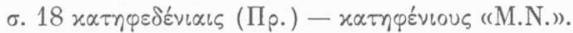

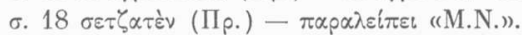

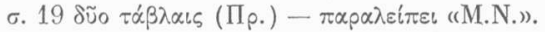

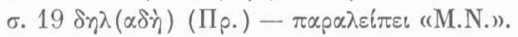

б. 20 है $\mu \alpha \gamma x \alpha \lambda_{l}\left(\Pi_{\rho}\right)$ - $\pi \alpha \rho \alpha \lambda \varepsilon i \pi \varepsilon \iota$ (M.N.).

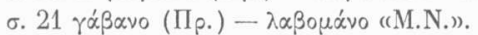

б. $28 \dot{\alpha} \xi i \alpha_{\varsigma}(\Pi p)-.\pi \alpha \rho \alpha \lambda \varepsilon i \pi \varepsilon !$ (M.N.).

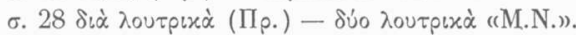

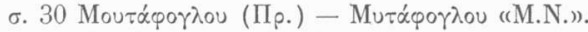

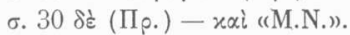

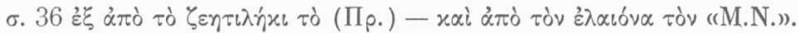

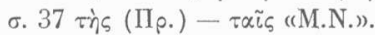

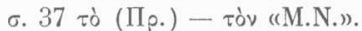

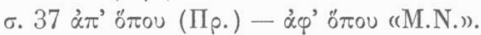

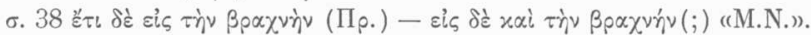

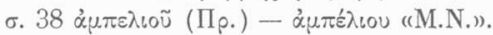

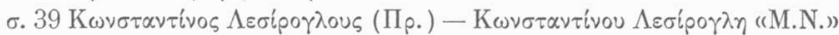

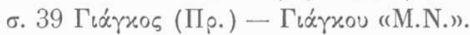

б. $40 \mathrm{~K} \varepsilon \mu \pi \alpha \nu \tilde{\alpha}_{\varsigma}(\Pi \rho)-.\mathrm{K} \varepsilon \lambda \tau \sigma \alpha \nu i \tilde{\alpha} \varsigma$ (M.N.).

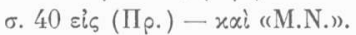

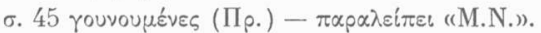

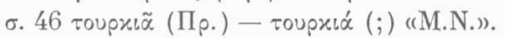

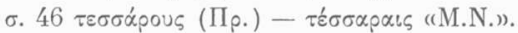

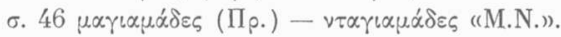




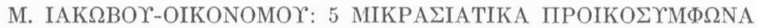

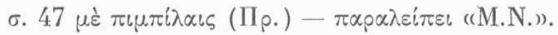

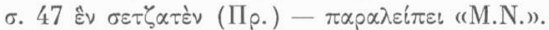

$\sigma .48 \mu \tilde{\imath} \alpha \mu \dot{\varepsilon} \alpha \tau \lambda \dot{\alpha} \zeta_{\zeta}(\Pi p)-.\mu i \alpha \mu \varepsilon \tau \alpha \xi \omega \tau \dot{\eta}$ (M.N.).

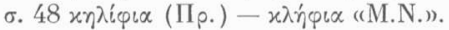

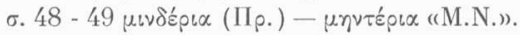

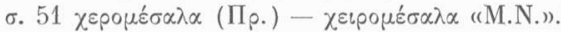

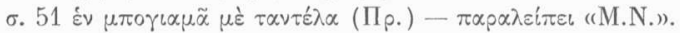

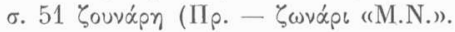

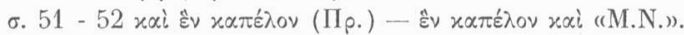

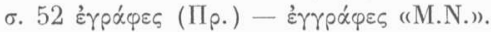

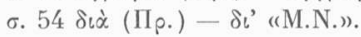

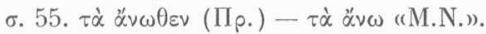

б. $55 \hat{\omega} \varsigma, \pi \rho \circ \sigma \theta \dot{\varepsilon} \tau \varepsilon \iota$ (M.N.).

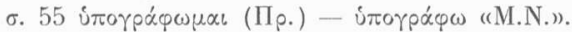

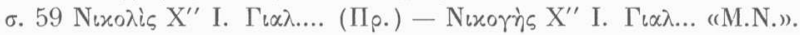

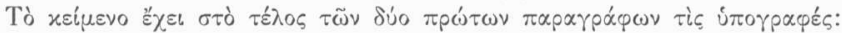

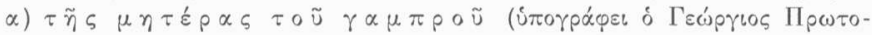

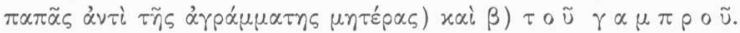

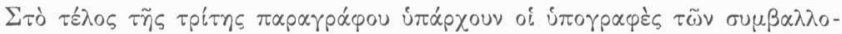

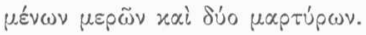

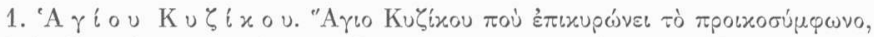

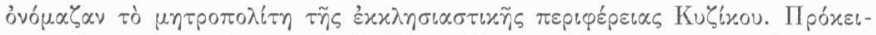

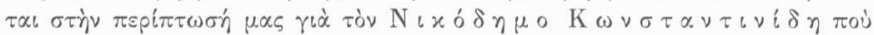

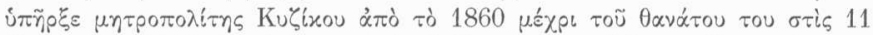

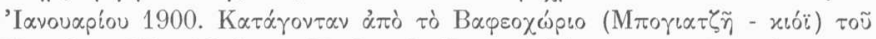

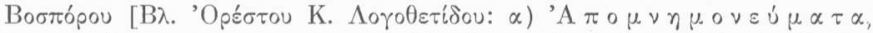

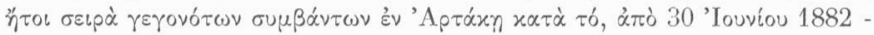

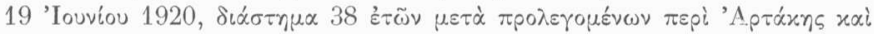

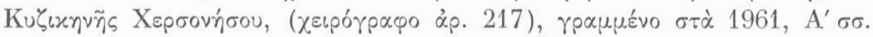

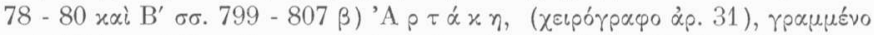

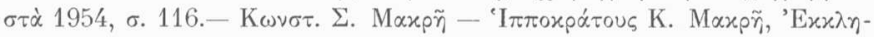

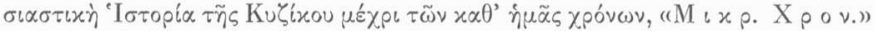

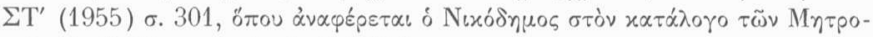

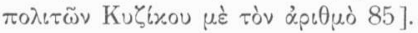

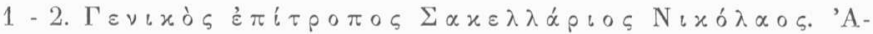

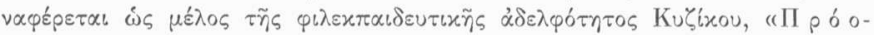

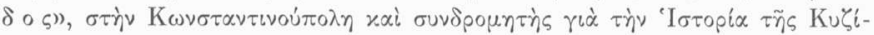

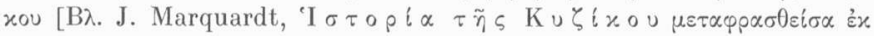




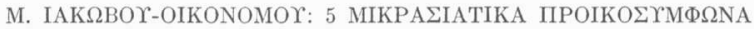

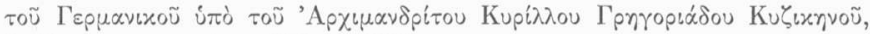

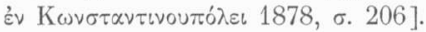

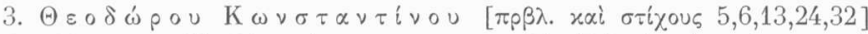

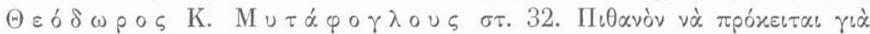

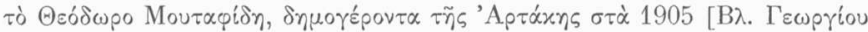

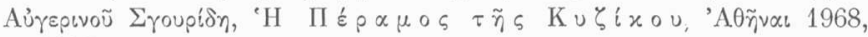
б. 222].

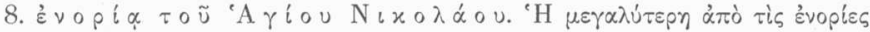

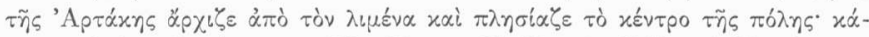

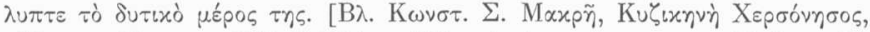

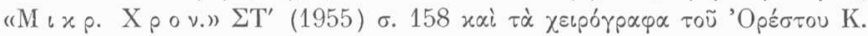

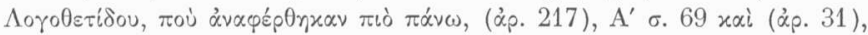
б. 111].

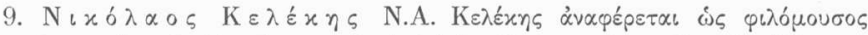

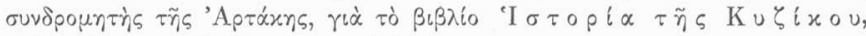

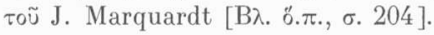

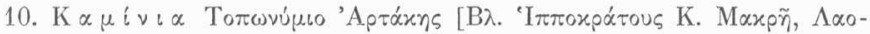

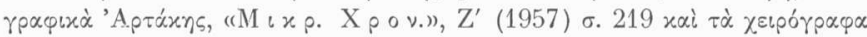

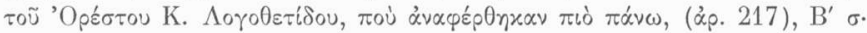

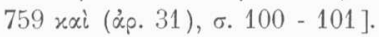

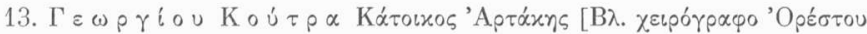

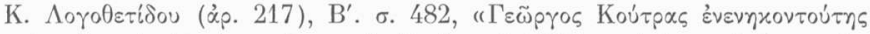

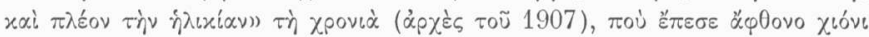

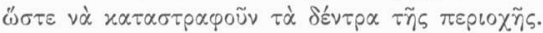

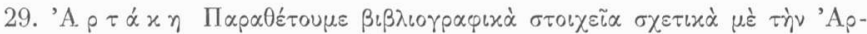

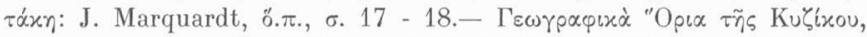

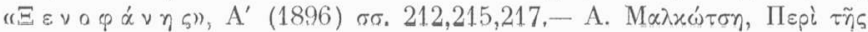

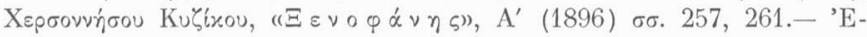

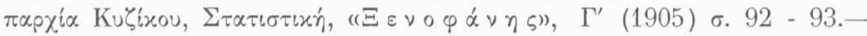

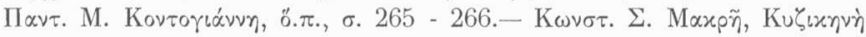

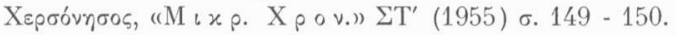

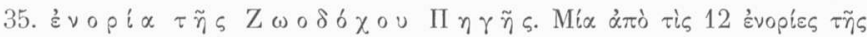

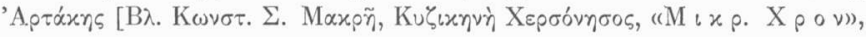




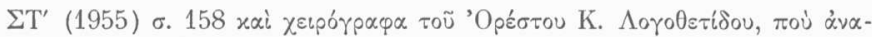

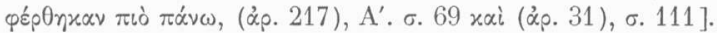

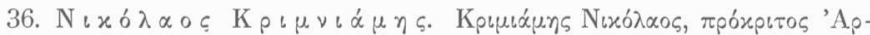

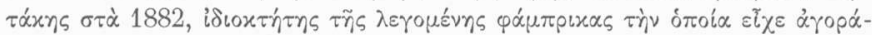

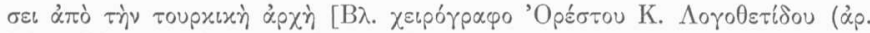

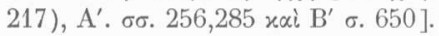

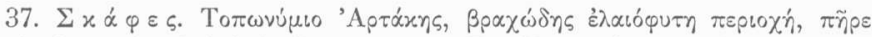

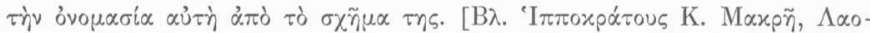

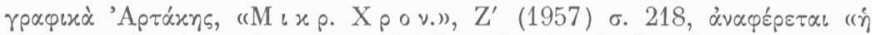

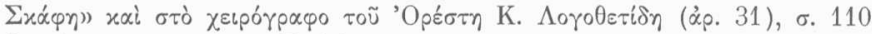

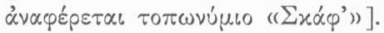

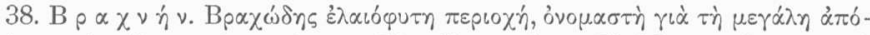

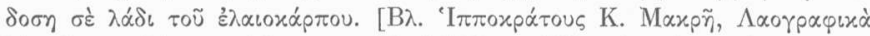

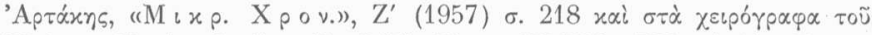

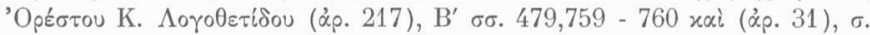
109.

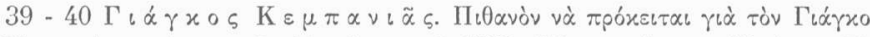

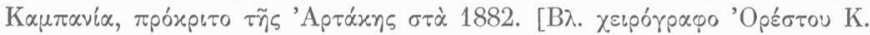

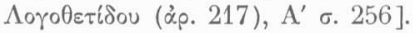

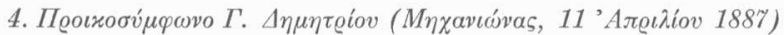

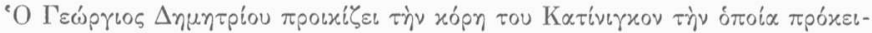

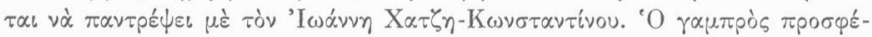

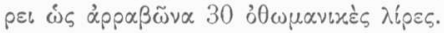

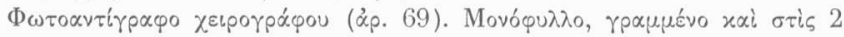

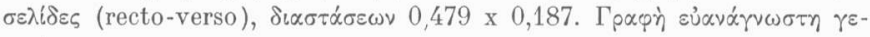
vเหน́.

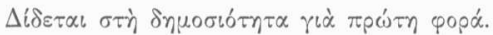

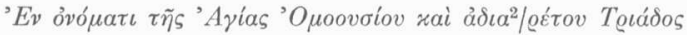

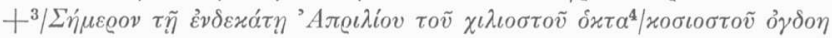

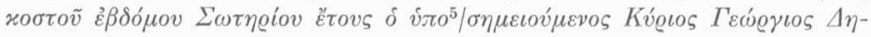

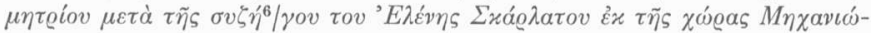

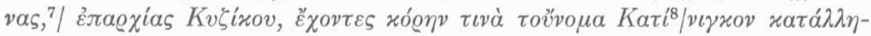




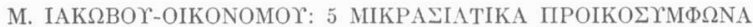

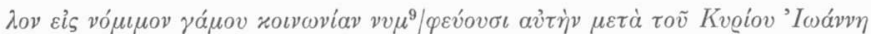

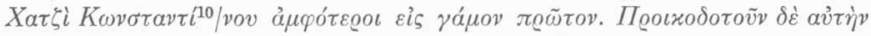
$11 / \delta \iota \dot{\alpha} \tau \tilde{\omega} v \varepsilon \xi \tilde{\xi} \zeta:$

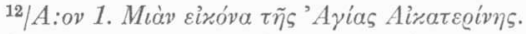

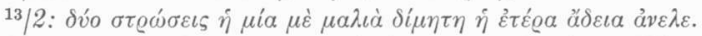

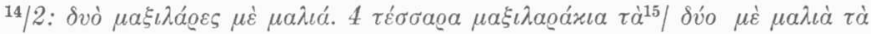

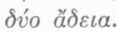

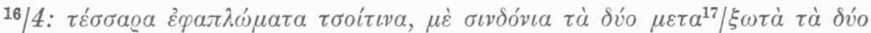
غ่vนóлıа...

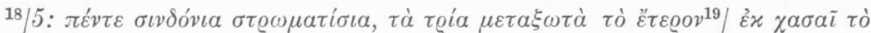

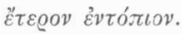

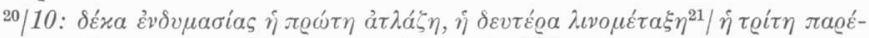

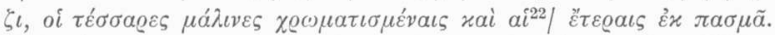

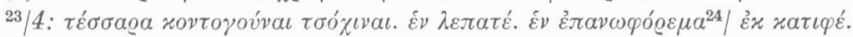

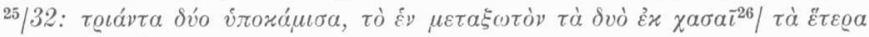

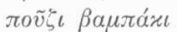

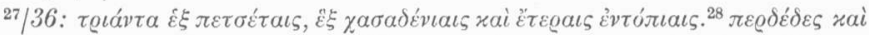

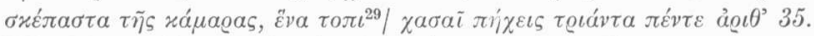

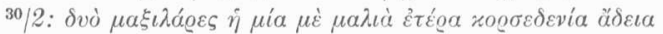

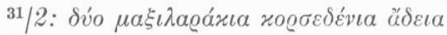

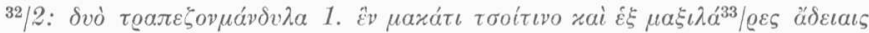
$\tau \sigma o i ́ \tau \iota \nu \varepsilon \varsigma$.

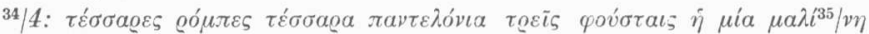

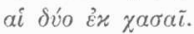

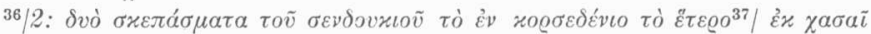

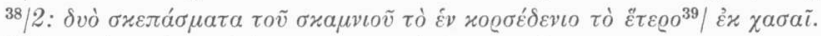

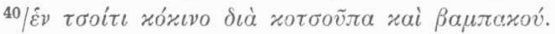

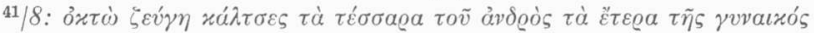

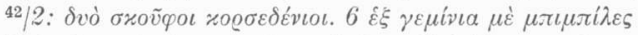

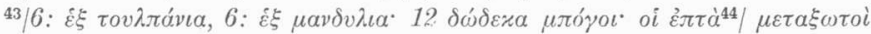
ai हैं $\varepsilon \varrho \alpha \iota \varsigma$ ह่x $\pi \alpha \sigma \mu \tilde{\alpha}$.

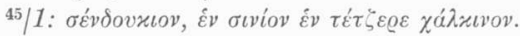

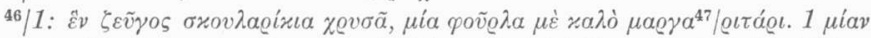

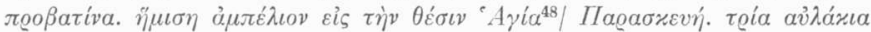

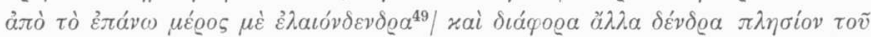

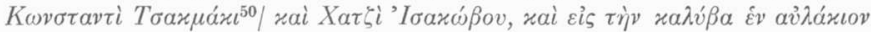

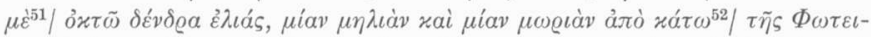

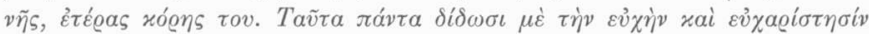

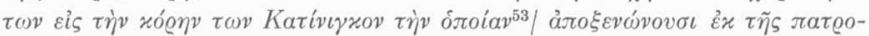

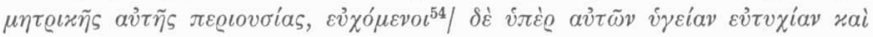




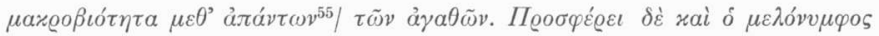

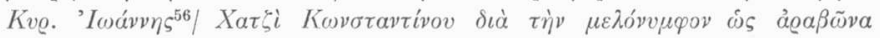

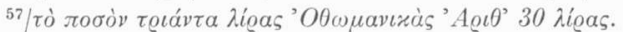

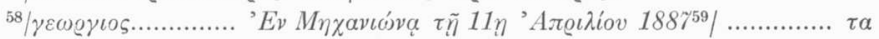

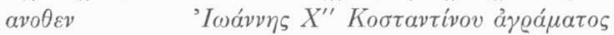

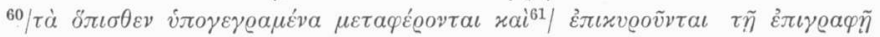
$\tau \tilde{\omega} \nu$ dे

63/ 'H 'Epoeía

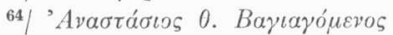

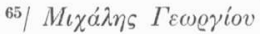

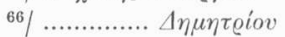

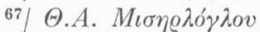

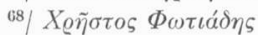

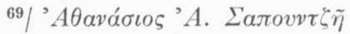

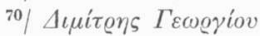

71

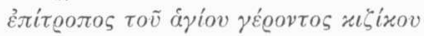

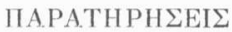

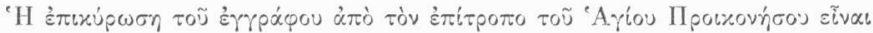

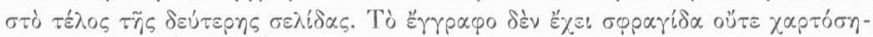

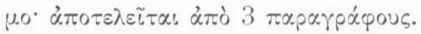

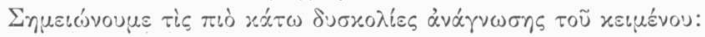

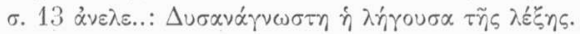

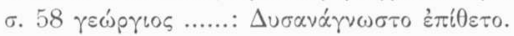

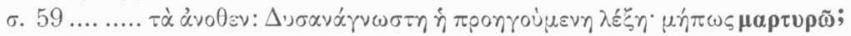

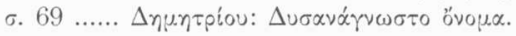

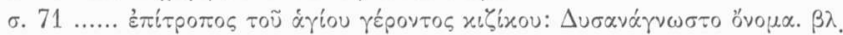
$\pi \alpha \rho \alpha \tau$.

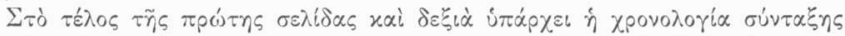

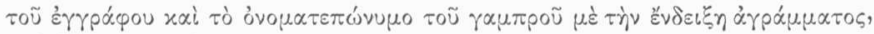

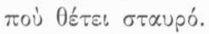

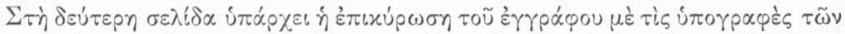

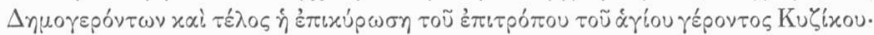

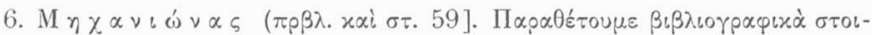

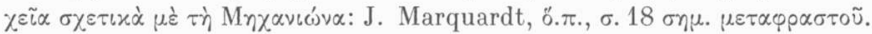

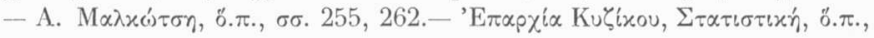




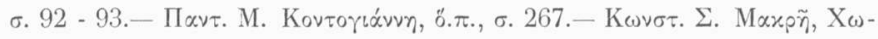

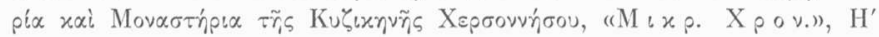
(1959) б. 152].

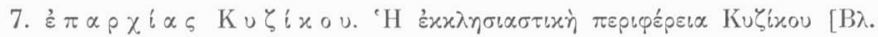

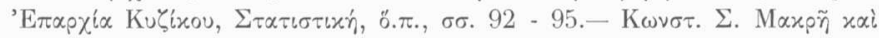

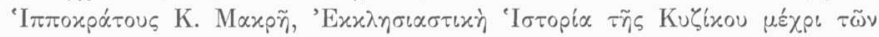

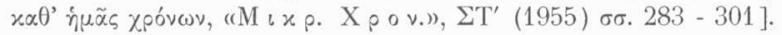

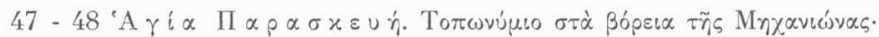

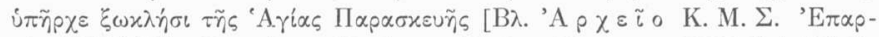

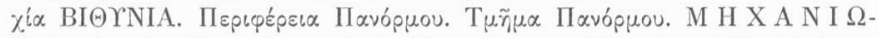

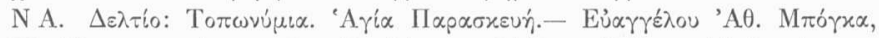

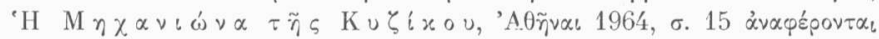

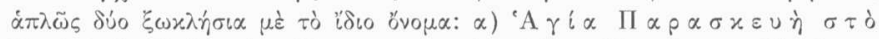

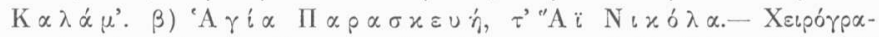

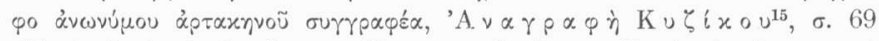

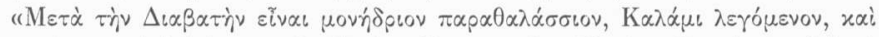

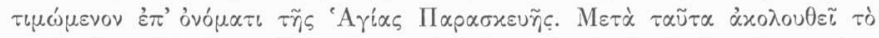

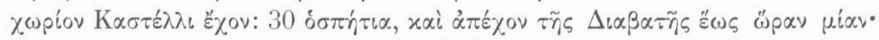

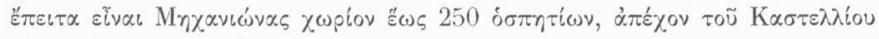

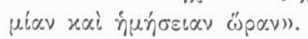

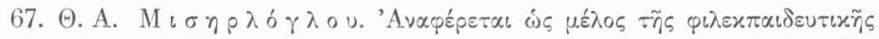

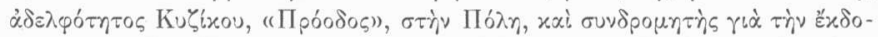

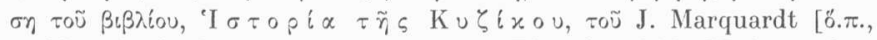

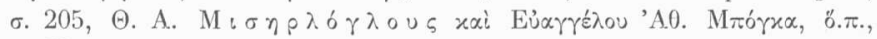
$\sigma \cdot 32]$.

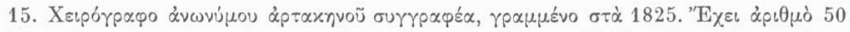

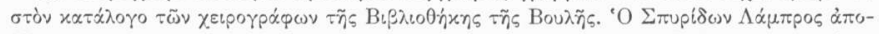

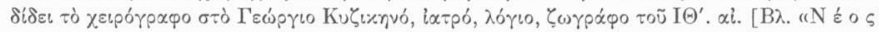
$\mathrm{E} \lambda \lambda \eta \nu \circ \mu \nu \dot{\eta} \mu \omega \nu), \mathrm{A}^{\prime}(1904) \sigma \sigma .72-88$ ].

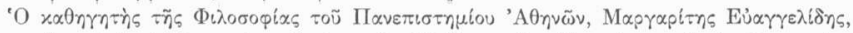

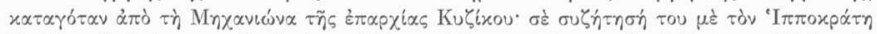

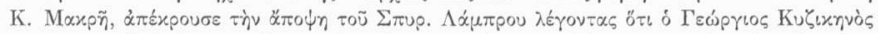

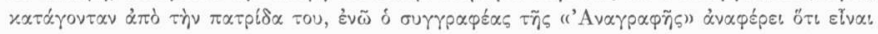

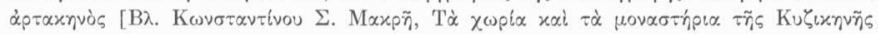

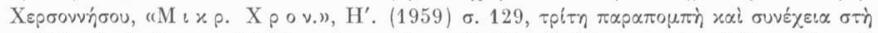

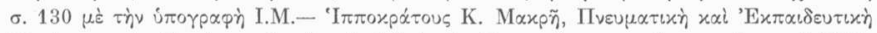

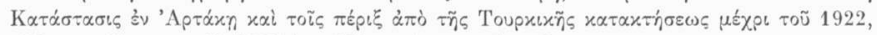

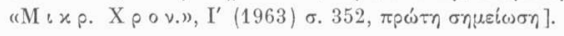




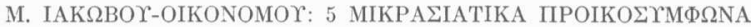

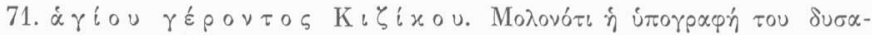

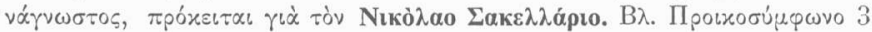

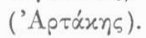

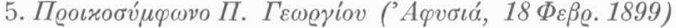

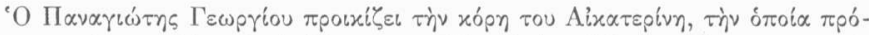

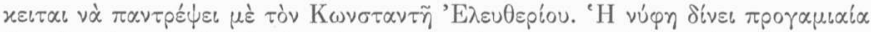

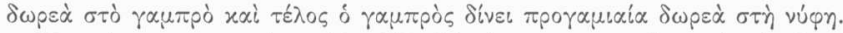

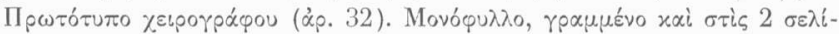

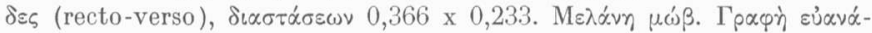
$\gamma \nu \omega \sigma \tau \eta . \Delta\llcorner\alpha \tau \eta p \varepsilon i \tau \alpha \iota$ бغे $\varkappa \alpha \lambda \dot{\eta}$ $x \alpha \tau \dot{\alpha} \sigma \tau \alpha \sigma \eta$.

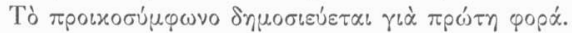

\section{Eis $\Lambda o ́ \xi \alpha v \Theta \varepsilon o \tilde{v}$}

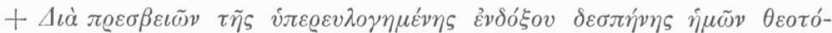

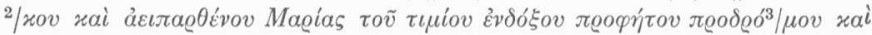

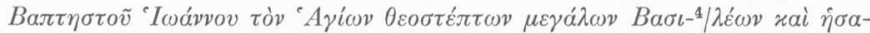

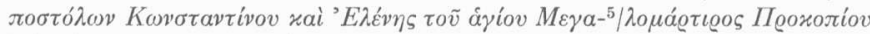

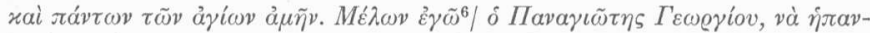

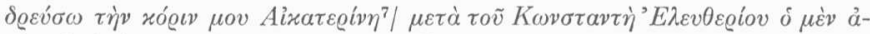

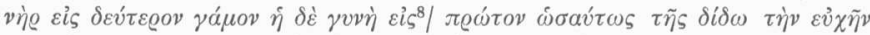

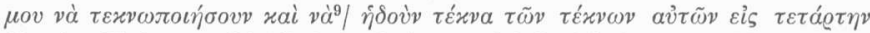

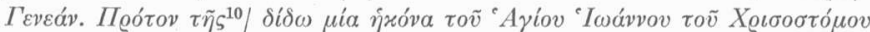

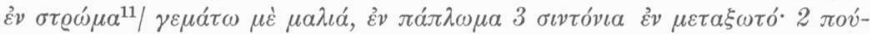

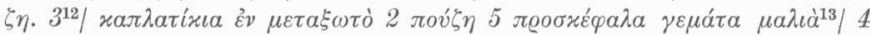

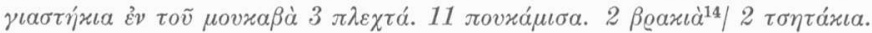

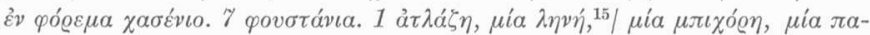

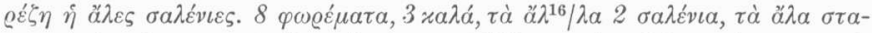

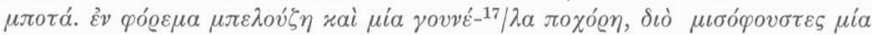

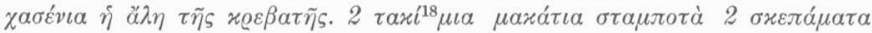

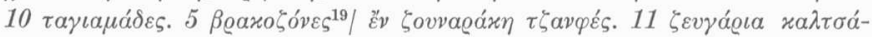

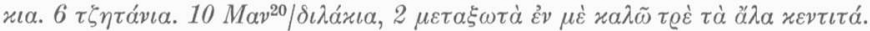

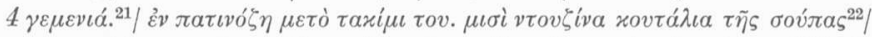

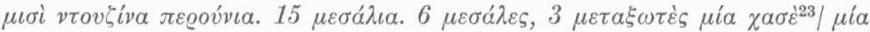

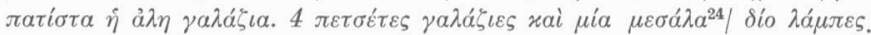

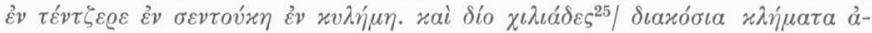

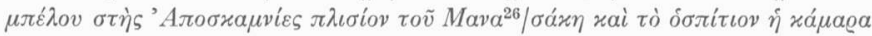

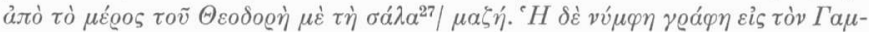

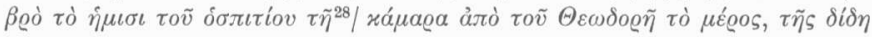




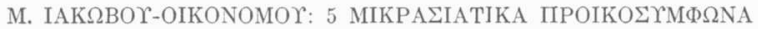

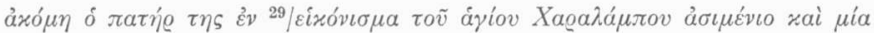

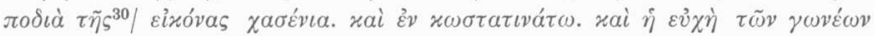

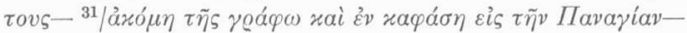

${ }^{32} /^{\circ} O \Sigma v \mu \beta \alpha \lambda o ́ \mu \varepsilon v o \varsigma$

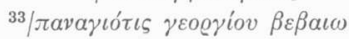

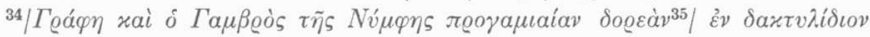

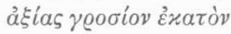

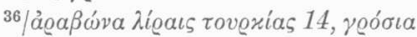

$\dot{\alpha} \circ \iota \mu \dot{\partial} \varsigma$

100

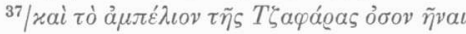

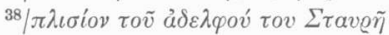

1.500

\section{${ }^{39} / \delta$ ov $\sigma \nu \beta \alpha \lambda{ }^{\prime} \mu \varepsilon v o s$

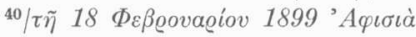

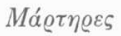

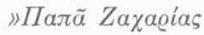

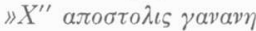

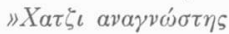

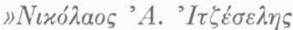

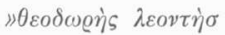

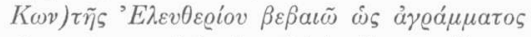

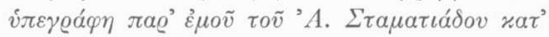

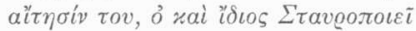

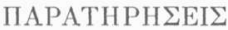

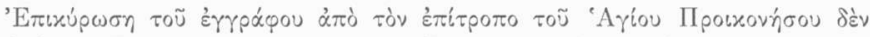

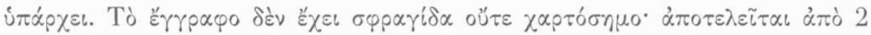

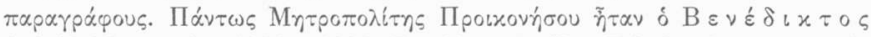

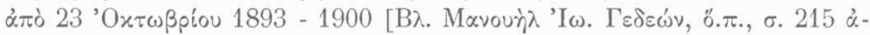

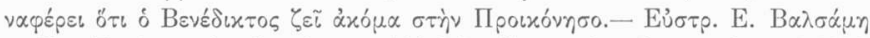

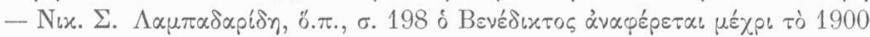

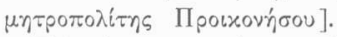

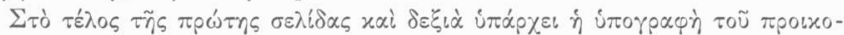

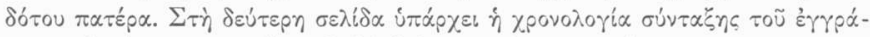

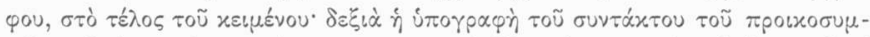

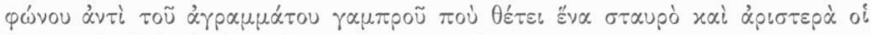

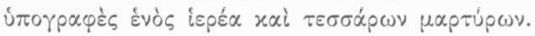

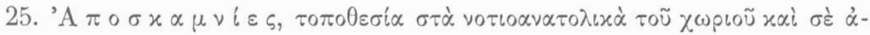

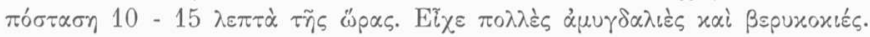

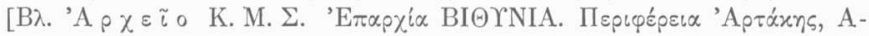

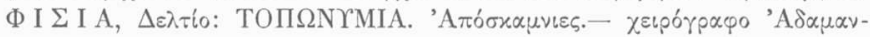

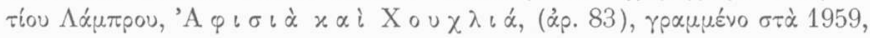

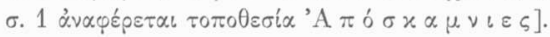




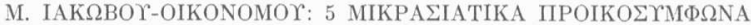

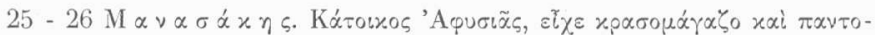

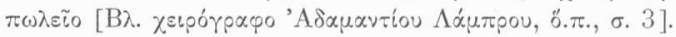

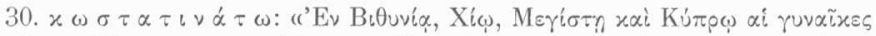

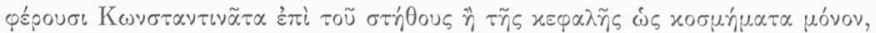

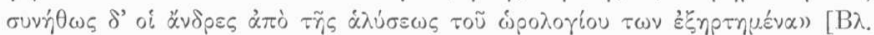

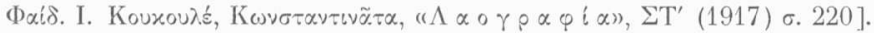

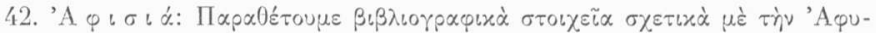

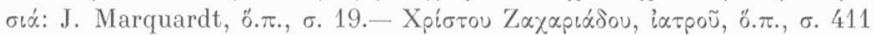

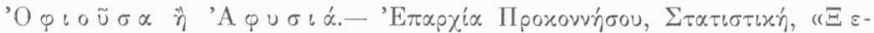

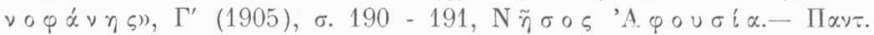

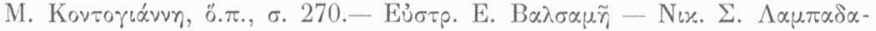

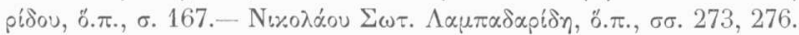

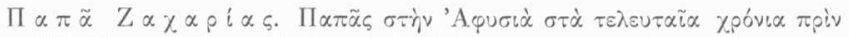

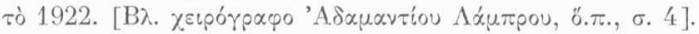

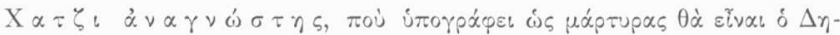

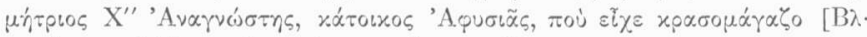

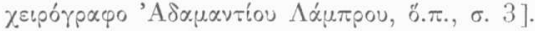

\section{$\Lambda \mathrm{E} \Xi \mathrm{I} \Lambda \mathrm{OIO} \mathrm{O}^{16}$}

$\dot{\alpha} \eta \nu \alpha \lambda \iota \dot{\alpha} \quad(\tau \dot{\alpha}) 3.25,28^{17} \quad\left(\varphi \lambda_{0}-\right.$ $\rho \imath \tilde{\alpha} \quad \dot{\alpha} v \alpha \lambda \iota \dot{\alpha})^{18} \beta \lambda . \phi \lambda \omega \rho\llcorner\dot{\alpha}$. $\dot{\alpha} \tau \lambda \dot{\alpha} \zeta \iota(\tau \grave{\jmath}) 2.17-.3 .48-.4 .20$ -

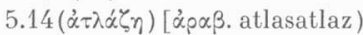

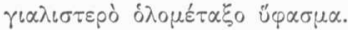

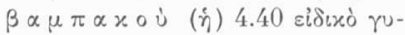

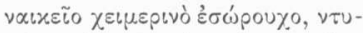

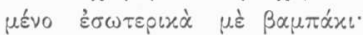

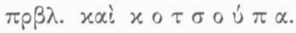

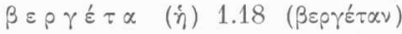
$[\mu \sigma \nu . \beta \varepsilon p \gamma \varepsilon \dot{\varepsilon} \tau \tau \alpha>i \tau \alpha \lambda$. verghet-

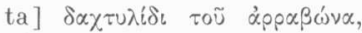
$\beta \varepsilon ́ p \alpha$.

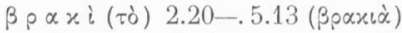

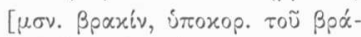

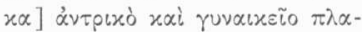

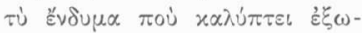

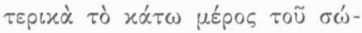

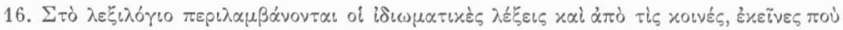

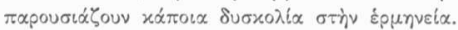

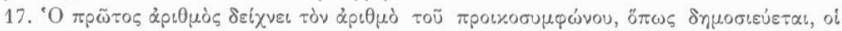

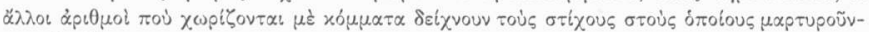
$\tau \alpha t$ oi $\lambda e^{\prime} \xi_{\varepsilon} \varepsilon \zeta$.

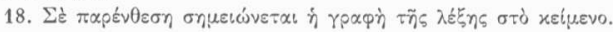




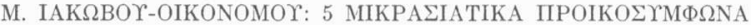

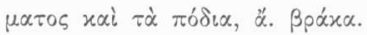
$\beta \rho \alpha x \circ \zeta \omega \nu \alpha(\dot{\eta}) 3.19,43-.5,19$

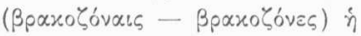

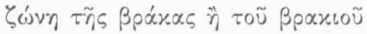

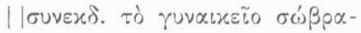
xo.

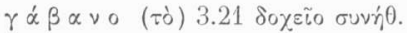

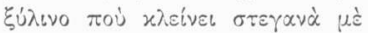

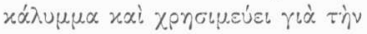

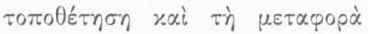

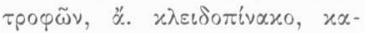
$\sigma \tau \alpha \nu เ \alpha \dot{ }$.

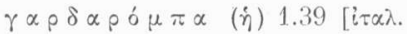
guarda-roba] है $\pi \iota \pi \lambda_{0} \mu \dot{\varepsilon}$ бup-

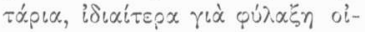

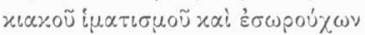

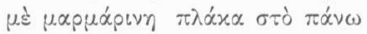

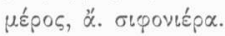

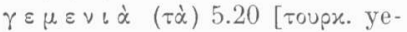

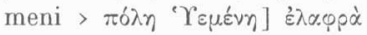

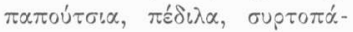

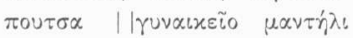
$\tau \tilde{\eta} \varsigma \quad x \varepsilon \varphi \alpha \lambda \tilde{\gamma} \varsigma \quad \pi \rho \beta \lambda . \quad \gamma \varepsilon \mu i \nu$ i.

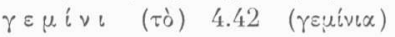

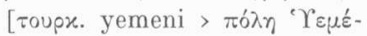

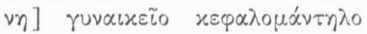

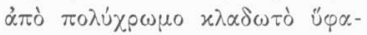
$\sigma \mu \alpha$.

$\gamma \iota \alpha \sigma \mu \dot{\alpha} \varsigma$ (o) $3.46 \quad(\gamma\llcorner\alpha \sigma \mu \alpha \dot{\alpha} \delta \varepsilon \varsigma)$

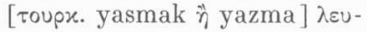

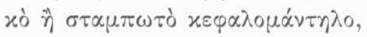
фаxเó̀t.

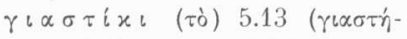

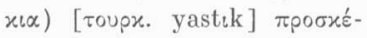
$\varphi \alpha \lambda_{0}, \mu \alpha \xi \iota \lambda \alpha \rho$.

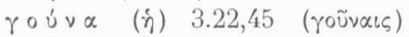

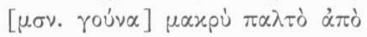

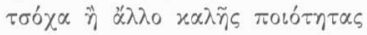

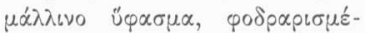

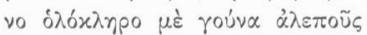

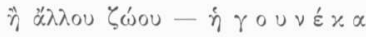

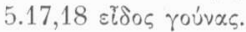

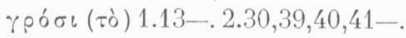
$3,28,29 \cdot 5,37,38 \quad(\gamma p(o ́ \sigma \iota)$

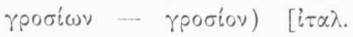

grosso > $\mu \sigma \nu . ~ \lambda \alpha \tau \imath$. (denarius

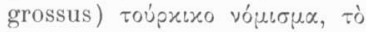

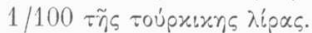

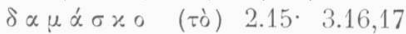

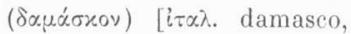

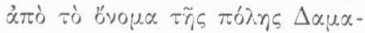

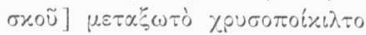

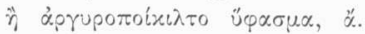
$\delta \alpha \mu \alpha \sigma \% \eta$ ขó.

$\delta$ ¿ $\mu \iota \tau \circ \varsigma_{-n}-0 \quad 2.11,13,25 \cdot 4.13$

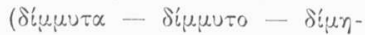

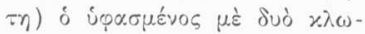

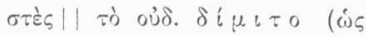

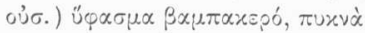
јџаб

$\delta เ \pi \lambda \circ \tilde{u} \varsigma \quad$ -

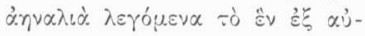

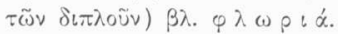

$\grave{\varepsilon} \gamma \rho \dot{\alpha} \varphi \alpha$ (i்) $x$. है $\gamma x \rho \dot{\alpha} \varphi \alpha x$.

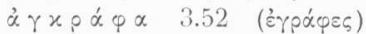

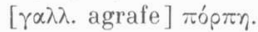

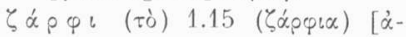
paß. zarf] $\theta \dot{n} x \eta \eta$

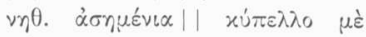

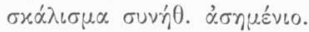

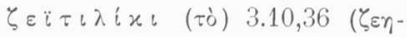

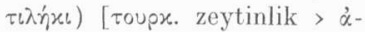

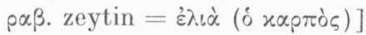

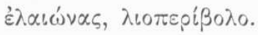

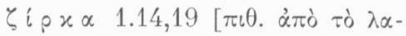

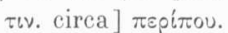

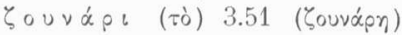

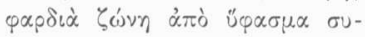

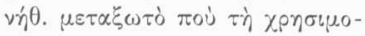

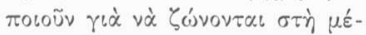




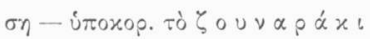

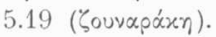

$x \alpha \zeta \dot{\alpha} x \alpha \quad(\dot{\eta}) \quad 1.28 \quad(x \alpha \zeta \alpha \dot{\alpha} x \alpha ! \zeta)$

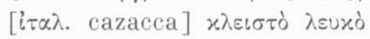

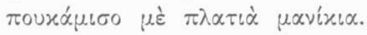
$x \alpha \lambda \varepsilon \mu x \varepsilon \rho i \quad(\tau \grave{o}) \quad 3.46 \quad(x \alpha-$ $\lambda \varepsilon \mu x \varepsilon p \alpha i \varsigma) \quad[\pi \varepsilon \rho \sigma$. Kalemkar: [тoupx. Kalemkarlik $=\dot{\eta} \beta \alpha$ -

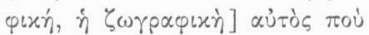

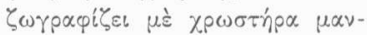

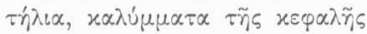

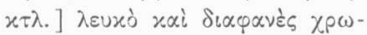

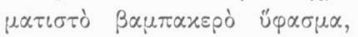

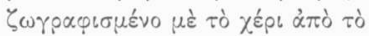

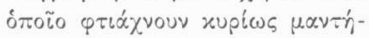
$\lambda\left\llcorner\alpha \tau \tilde{\eta} \varsigma \quad x \varepsilon \varphi \alpha \lambda \tilde{r}_{i} s\right.$.

$x \alpha \pi \alpha x \lambda i \delta\llcorner x \circ \leqslant-\eta \quad-0 \quad 3.21$ $(x \alpha \pi \alpha x \lambda i \delta \iota x \alpha)$ [тоupx. kapa-

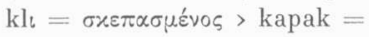
$\left.\sigma x \varepsilon ́ \pi \alpha \sigma \mu \alpha, \alpha_{\alpha}^{\prime} \lambda u \mu \mu \alpha\right] \mu \dot{~} \chi \alpha \pi \alpha$ $x$.

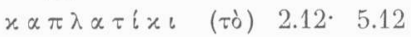

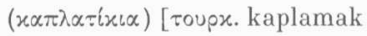
p. $\mu \tau \beta .=$ ย่ $\pi \varepsilon v \delta \dot{v} \omega, \pi \varepsilon \rho \iota x \alpha \lambda \dot{u}-$

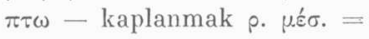

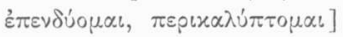

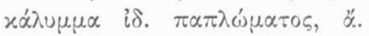

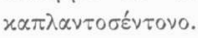

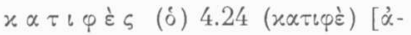

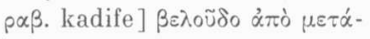

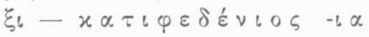

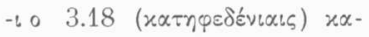

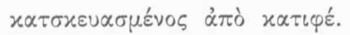

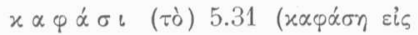

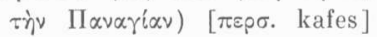

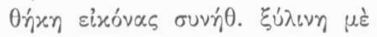

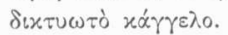

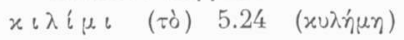

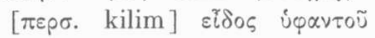

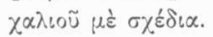

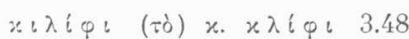

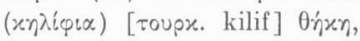

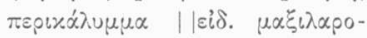
$\theta \dot{n} x \eta \eta$

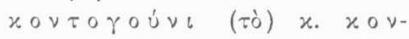

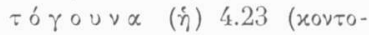

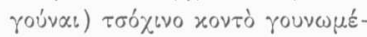

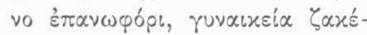

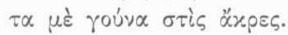

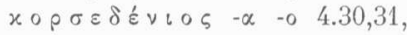

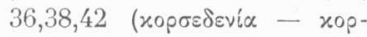

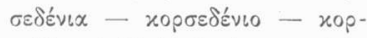

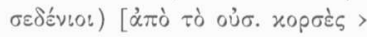

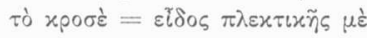

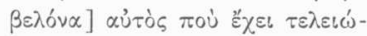

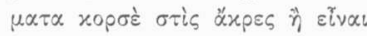

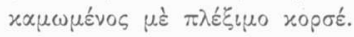

$x \circ \tau \sigma \circ \dot{u} \pi \alpha(\dot{\eta}) x . x \circ \cup \tau \sigma \circ \dot{u}-$

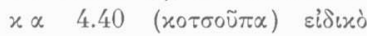

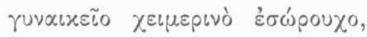

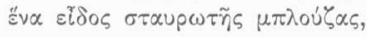

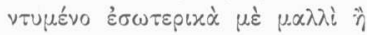
$\beta \alpha \mu \pi \alpha \dot{x}$.

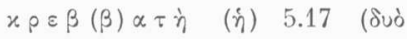

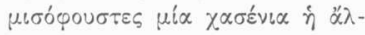

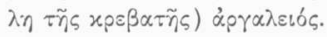

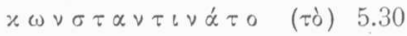

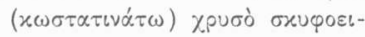

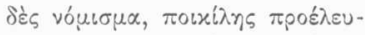

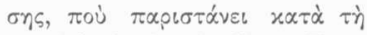
$\lambda \alpha \ddot{x} x \dot{~ \alpha े v \tau i ́ \lambda \eta \psi \eta ~ \tau o ̀ v ~ " A \gamma r o v ~ K \omega v-~}$

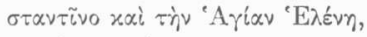
$\pi \circ \lambda \lambda \varepsilon$ दे

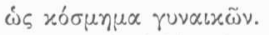

$\lambda \dot{\alpha} x x \circ \varsigma$ (o) 2.31 (рó $\mu \pi \varepsilon \varsigma$

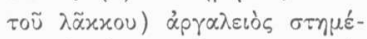

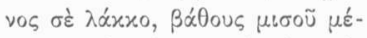

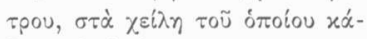
$\theta \varepsilon \tau \alpha \iota \dot{\eta} \dot{u} \varphi \alpha \dot{\alpha} \tau \rho \iota \alpha$.

$\lambda \varepsilon \gamma \varepsilon v$ ó $\mu . \pi \rho$ เ 


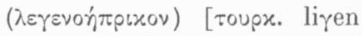
$\dot{\eta}$ le ren + ibrik] $\dot{\eta} \lambda \varepsilon x \alpha \dot{\alpha} \nu \eta$ xaì

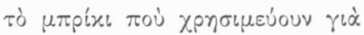

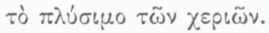

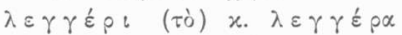

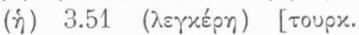
lenger] $\chi^{\alpha} \lambda x เ \nu \eta \mu \varepsilon \alpha^{\prime} \lambda \eta \eta \dot{\alpha} \beta \alpha-$ $\theta \dot{\eta} \bar{s} \pi\llcorner\alpha \tau \dot{c} \lambda \alpha$.

$\lambda \varepsilon \pi \alpha \tau \dot{\varepsilon} \zeta \quad(\dot{0}) \quad \chi . \lambda \iota \mu \pi \alpha \tau \dot{\varepsilon} \varsigma$

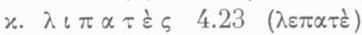
[roupr. libade $=\mu \alpha \nu \delta \dot{\delta} \alpha \varsigma>\dot{\alpha}$ -

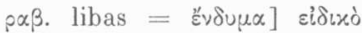

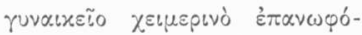

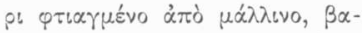

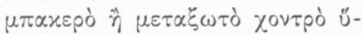

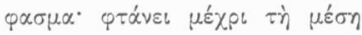

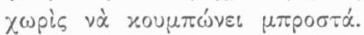
$\lambda i \rho \alpha \quad(\dot{\eta}) \quad 3.25,26,27,40^{\circ}$ 4.58.

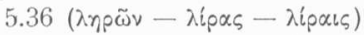

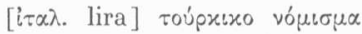

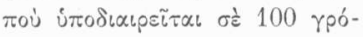
$\sigma: \alpha$.

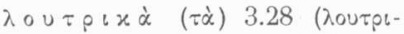

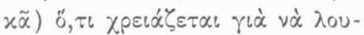

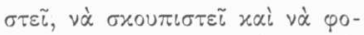

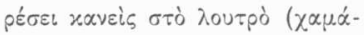
(ki).

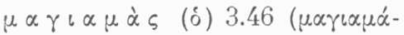
$\delta \varepsilon \varsigma)$;

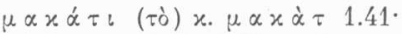
$2.25 \cdot 3.17,49 \cdot 4.32 \cdot 5.18$ ( $\mu \alpha-$

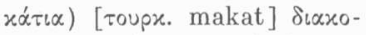

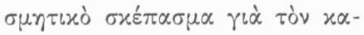

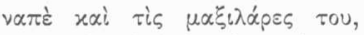

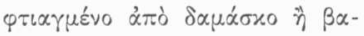
$\mu \pi \alpha x \varepsilon \rho \grave{~} \ddot{\varphi} \propto \sigma \mu \alpha$.

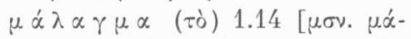
$\lambda \alpha \mu \alpha>\dot{\alpha} \rho \chi . \mu \alpha^{\prime} \lambda \alpha \gamma \mu \alpha>\mu \alpha \lambda \dot{\alpha} \sigma-$ $\sigma \omega] \mu \alpha \dot{\alpha} \lambda \alpha \mu \alpha, \chi p \cup \sigma \delta \zeta$.

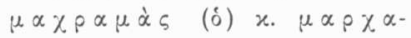

$\mu \dot{\alpha} \varsigma \quad 1.34 \quad(\mu \alpha \chi p \alpha \mu \alpha \dot{\delta} \delta \varsigma) \quad[\dot{\alpha}-$

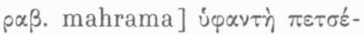

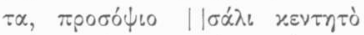

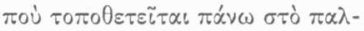
$\tau \dot{0}$.

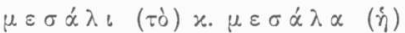
$2.23,24 \cdot 5.22,23 \quad(\mu \varepsilon \sigma \alpha \dot{\lambda} \varepsilon \varepsilon-$

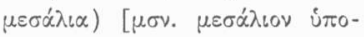

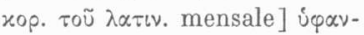

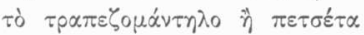

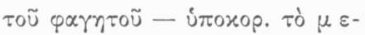
$\sigma \alpha \lambda \dot{\alpha} x \iota \quad 2.24 \quad(\mu \varepsilon \sigma \alpha \lambda \dot{\alpha} \alpha \iota \alpha$

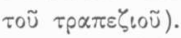

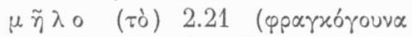

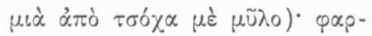
$\delta$ ¿े̀ xóxxivn roúva.

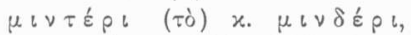
$\mu \varepsilon \tau \varepsilon \dot{\rho}$ เ 1.42. 2.27 3.17,

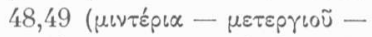

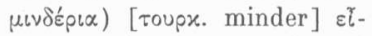

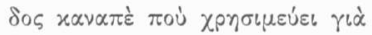
$\alpha \dot{\alpha} \alpha \dot{\alpha} \pi \alpha u \sigma \eta|| \sigma u v \varepsilon x \delta$. $\lambda \varepsilon \pi \tau \dot{\partial} \beta \alpha-$

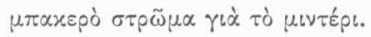
$\mu \iota \sigma o ́ \varphi \circ v \sigma \tau \alpha$ (ं் $-\mu \iota \sigma 0-$ $\varphi \circ u ́ \sigma \tau \alpha \nu \circ$ (tò) 1.223 .44

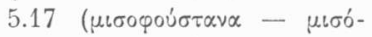

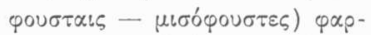

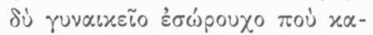

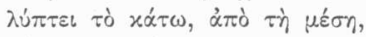

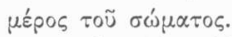

$\mu \circ \cup x \alpha \beta \dot{\alpha} \varsigma(\delta) 5.13(\mu \circ u x \alpha \beta \dot{\alpha})$

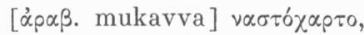

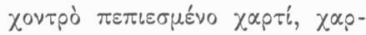
Tóve.

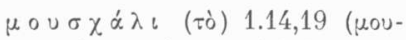

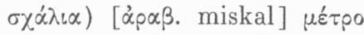

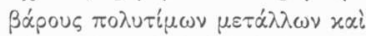

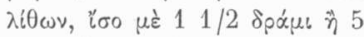

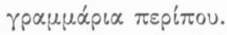

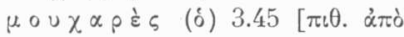


$\tau \dot{\alpha} \alpha \dot{\rho} \alpha \beta$. buhar $=\dot{\alpha} \tau \mu \alpha_{\zeta}, \dot{\alpha} \nu \alpha-$

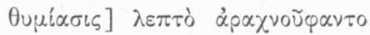
ü $\varphi \alpha \sigma \mu \alpha$.

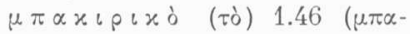
xupuxòv) [roupx. bakir] x. бu-

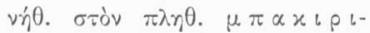
$x \dot{\alpha}, \tau \dot{\alpha} \chi \alpha \lambda x \dot{\omega} \mu \alpha \tau \alpha, \tau \dot{\alpha} \chi^{\alpha} \lambda x u-$ $v \alpha \sigma x \varepsilon \dot{u} \eta$

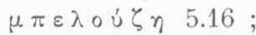

$\mu \pi \iota \mu \pi i \lambda \alpha$ (í) $4.42 \quad(\mu \pi \iota \mu \pi i-$

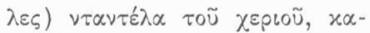

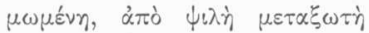

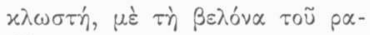

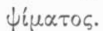

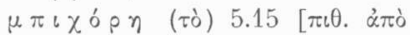

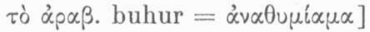

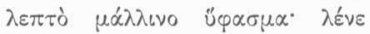

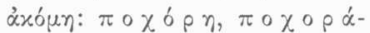
$x_{\iota} \pi \rho \beta \lambda . x \alpha i \quad \mu \circ \cup \chi \alpha \rho \varepsilon \xi$.

$\mu \pi \circ \gamma \iota \alpha \mu \dot{\alpha} s \quad(\delta) \quad 3.51 \quad(\mu \pi 0-$ $\gamma(\alpha \mu \tilde{\alpha})$; [roupx. boyama $=$

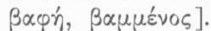

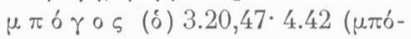

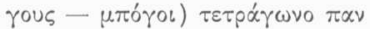

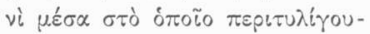

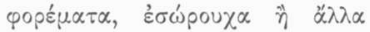

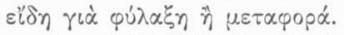

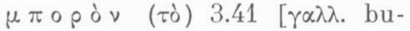

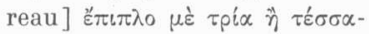

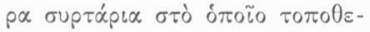

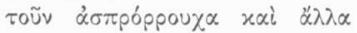

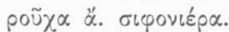

$\mu \pi \circ \chi \tau \zeta \dot{\alpha} \sigma \quad(\delta) \quad x . \mu \pi \circ \xi \dot{\alpha} \varsigma$

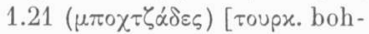

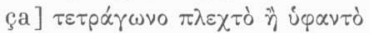

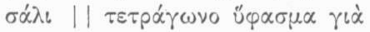

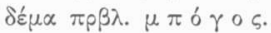

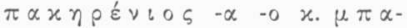

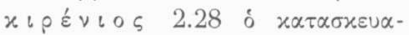

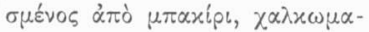

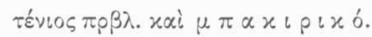

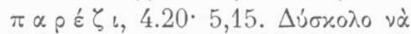

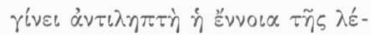

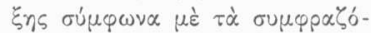
$\mu \varepsilon v \alpha \cdot \pi \alpha \dot{\alpha} \nu \tau \omega \varsigma \dot{\eta} \lambda$. .

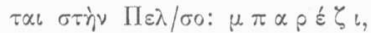

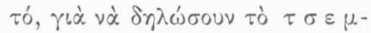

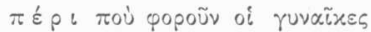

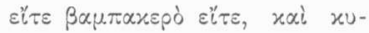

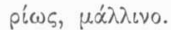

$\pi \alpha \sigma \mu \grave{\alpha} \varsigma \quad$ (ó) $2.19,26 \cdot \quad 4.22,44$

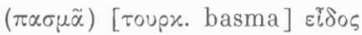
$\beta \alpha \mu \pi \alpha x \varepsilon \rho о \tilde{~} \dot{\varphi} \alpha \dot{\alpha} \sigma \alpha \alpha \tau \sigma \zeta$ $\mu \dot{\varepsilon} \tau-$

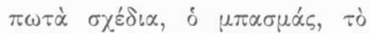

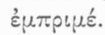

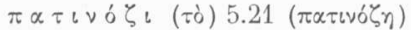

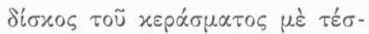
$\sigma \varepsilon \rho \alpha \mu l x \rho \dot{\alpha} \quad \pi 0 \delta \alpha \rho \dot{\alpha} x \iota \alpha$ $\gamma(\dot{\alpha} \quad \nu \dot{\alpha}$

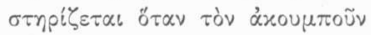
xár

$\pi \alpha \tau i \sigma \tau \alpha$ (i) $5.23[\gamma \alpha \lambda \lambda$. ba-

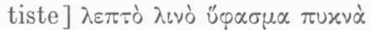

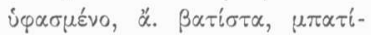
$\sigma \tau \alpha$.

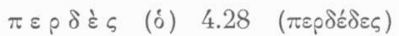

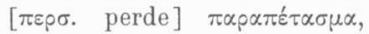

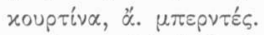

$\pi \iota \mu \pi i \lambda \alpha \quad(\dot{\eta}) 3.47 \quad(\pi \imath \mu \pi i \lambda \alpha \iota \zeta)$

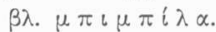

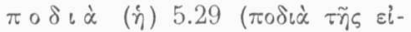

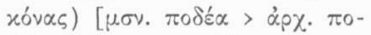

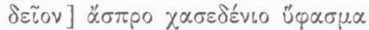

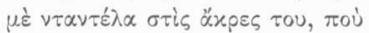

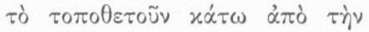

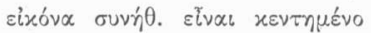

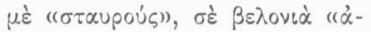
לoúp)".

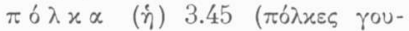

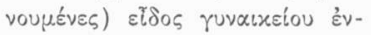

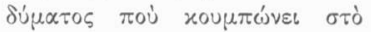




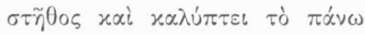

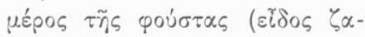

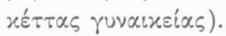

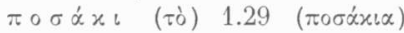

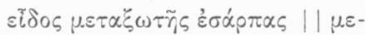
$\gamma \alpha \dot{\alpha} \lambda_{0} \not ้ \sigma \pi \rho \circ \quad \mu \varepsilon \tau \alpha \xi \omega \tau o ̀ ~ \mu \alpha \nu \tau \dot{\eta} \lambda_{l}$. $\pi$ oú $\zeta_{6} 4.25 \cdot 5.11,12$;

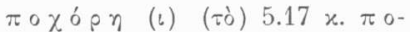

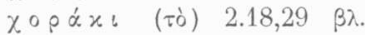

$$
\begin{aligned}
& \mu \pi \iota \chi \dot{\alpha} \rho \eta \text {. }
\end{aligned}
$$

pó $\pi \propto$ (并) $1.20 \cdot 2.30 \cdot 3.44 \cdot 4.34$

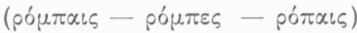
$[i \tau \alpha \lambda$. roba > $\alpha \rho \chi . \gamma \varepsilon p \mu$. rou-

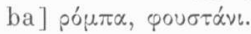

$\sigma \alpha \lambda \varepsilon \vee \iota 0 \varsigma \quad-\alpha \quad-0 \quad 5.15,16 \quad(\sigma \alpha-$

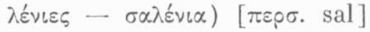

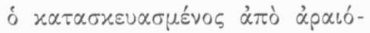

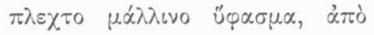
$\sigma \alpha \dot{\lambda}$.

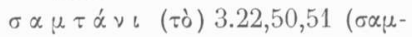
$\tau \alpha \dot{v} \nu \alpha-\sigma \alpha \mu \tau \alpha \dot{v} v \varepsilon \iota \alpha) \quad[\pi \varepsilon \rho \sigma$.

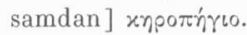

$\sigma \alpha \chi \alpha \dot{\alpha} \vee \quad(\tau \dot{0}) \quad 3.21 \quad(\sigma \alpha \chi \alpha \dot{\alpha} \nu\llcorner\alpha)$

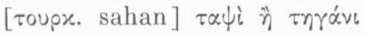

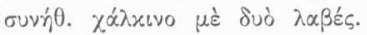

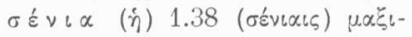
$\lambda \alpha p o \theta \dot{n} x \eta$.

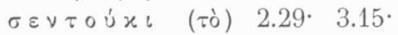

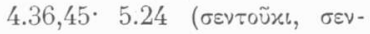

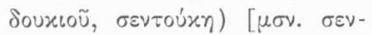

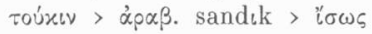

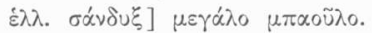

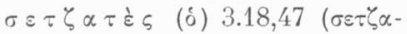

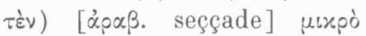

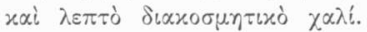

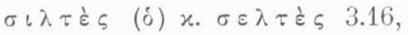

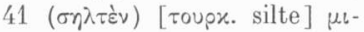

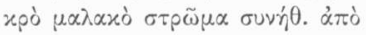
$\beta \alpha \mu \pi \alpha \alpha_{\ell}|| \sigma \tau \rho \omega \mu \alpha \tau o ́ \pi \alpha \nu 0$ ||

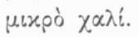

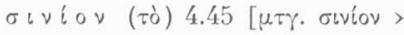

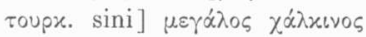

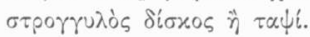

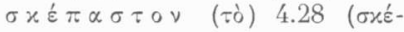

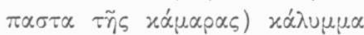

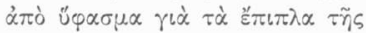
$x \rho \varepsilon \beta \beta \alpha \tau \sigma x \alpha \dot{\alpha} \mu \alpha \alpha \varsigma$.

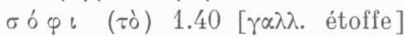

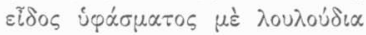

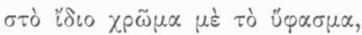
$\dot{\eta} \sigma \tau \dot{\varphi} \varphi \alpha$.

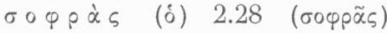

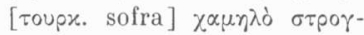

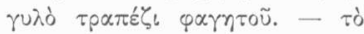
$\sigma \circ \varphi p \alpha \lambda i x i \quad 2.25 \quad(\sigma \circ \varphi p \alpha-$

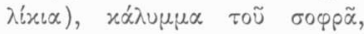
$\tau \rho \alpha \pi \varepsilon \zeta o \mu \alpha \dot{\alpha} \nu \tau \eta \lambda$.

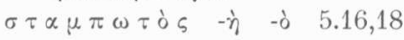

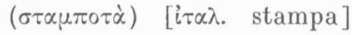

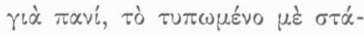
$\mu \pi \alpha$.

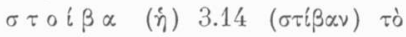

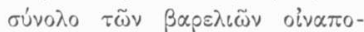
$\theta \dot{n} x$ s.

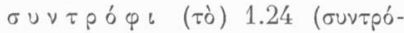

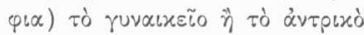

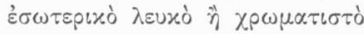

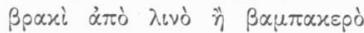
Ü $\varphi \alpha \sigma \mu \alpha$.

$\tau \dot{\alpha} \beta \lambda \alpha(\dot{\eta}) 3.19(\tau \dot{\alpha} \beta \lambda \alpha \iota \varsigma)[\mu \sigma \nu$. $\tau \dot{\alpha} \beta \lambda \alpha>\lambda \alpha \tau \iota v$. tab(u)la] $\sigma \tau \varepsilon-$

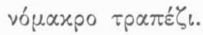

$\tau \alpha \gamma \iota \alpha \mu \alpha \varsigma$ (ó) $2.14 \cdot 3.18 \cdot 5.18$

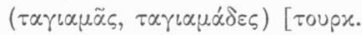
tagam $=\tau \circ \tilde{\iota} \chi \circ \varsigma$ $\ddot{\eta}$ dayamak p. $\mu \tau \beta .=\sigma \pi \eta \rho^{\prime \zeta \zeta} \omega, \dot{\alpha} x \circ u \mu \pi \tilde{\omega}$

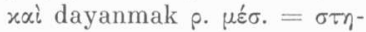

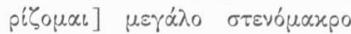

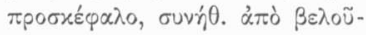

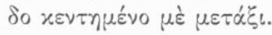


$\tau \alpha x\lfloor\mu \iota$ (т̀े) $2.26 \cdot 3.17,19,42$.

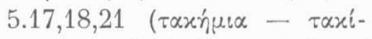

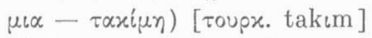

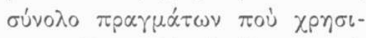

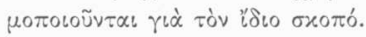
$\tau \alpha \pi \lambda \dot{\alpha} \varsigma \quad(\dot{\delta}) 3.20(\tau \alpha \pi \lambda \tilde{\alpha} \nu)$ [ $\dot{\alpha}$ -

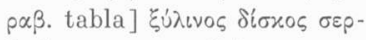
$\beta \iota p i \sigma \mu \alpha \tau \sigma \zeta, \gamma . \tau \alpha \beta \lambda \alpha \dot{s}$.

$\tau \zeta \alpha \nu \varphi \grave{\varepsilon} \varsigma \quad(\delta) \quad x . \tau \zeta \alpha \mu \varphi \grave{\varepsilon} \zeta$

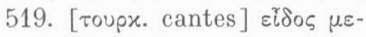
$\tau \alpha \hat{\xi} \omega \tau 0 \tilde{u}$ í $\dot{\alpha} \sigma \mu \alpha \tau \sigma \varsigma, \tau \alpha \varphi \tau \dot{\alpha} \zeta$

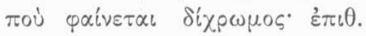

$\tau \zeta \alpha \mu \varphi \varepsilon \delta \varepsilon \nu \iota 0 \zeta \quad-\imath \alpha \quad-\iota 0$

$3.45(\tau \zeta \alpha \mu \varphi \varepsilon \delta \varepsilon \dot{v} \alpha \alpha)$ i $x \alpha \tau \alpha-$

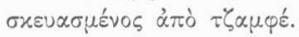

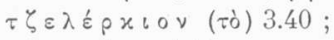

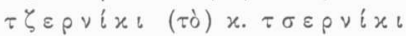

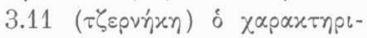

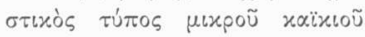

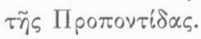

$\tau \zeta \eta \tau \dot{\alpha} \nu \iota \alpha \quad(\tau \dot{\alpha}) 5.19$;

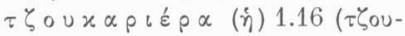

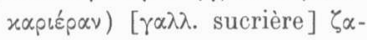

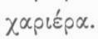

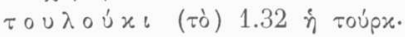

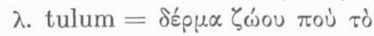

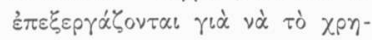

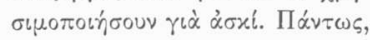

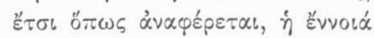

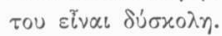

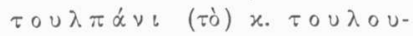
$\pi \dot{\alpha} \nu: 4.43(\tau 0 \nu \lambda \pi \dot{\alpha} v L \alpha)[\mu \sigma \nu$.

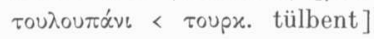

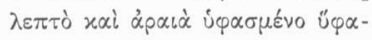
$\sigma \mu \alpha|| \sigma u \nu \varepsilon x \delta$. $x \varepsilon \varphi \alpha \lambda o \mu \alpha \dot{\alpha} \tau \eta \lambda 0$,

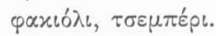

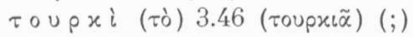

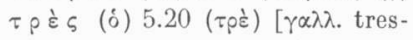
se] $\mu \alpha \lambda \alpha \mu \alpha \tau \sigma \alpha \alpha \alpha \tau \nu \sigma \mu \varepsilon^{\prime} \eta \eta \mu \varepsilon \alpha_{\alpha} \lambda-$ $\lambda เ v \eta \pi 0 \lambda \dot{u} \sigma \tau \varepsilon v \dot{\eta}$ $\alpha \dot{\alpha} \lambda \varepsilon \varepsilon \pi \dot{\eta} \tau \alpha \iota-$

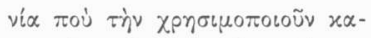

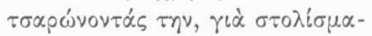

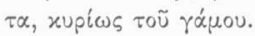

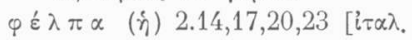

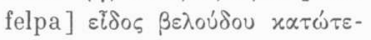

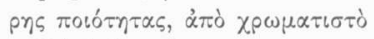
$\mu \alpha \lambda \lambda i$ में $\beta \alpha \mu \pi \alpha \dot{x} x$.

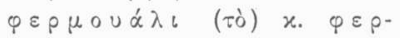

$\mu \circ \cup \dot{\alpha} \gamma \iota 1.19 \quad[\pi t \theta . \quad \gamma \alpha \lambda \lambda$.

fermoir] $\gamma$

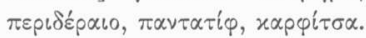

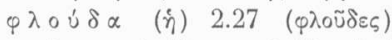

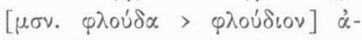

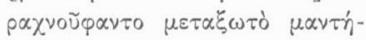
$\lambda_{l} \tau \tilde{\eta} \zeta \quad x \varepsilon \varphi \alpha \lambda \tilde{n} s$.

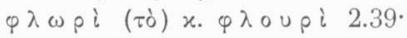
3.25,28 ( $\varphi \lambda \omega \rho\llcorner\dot{\alpha}-\varphi \lambda . o p l \tilde{\alpha} \alpha \dot{\alpha} \eta$

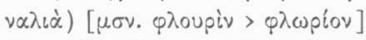

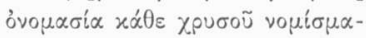
$\tau \circ \varsigma|| \dot{\alpha} \eta \nu \alpha \lambda เ \dot{\alpha} \quad[\pi t \theta$. $\alpha \pi \dot{o}$

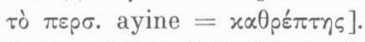

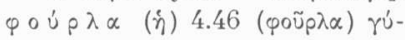

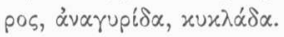

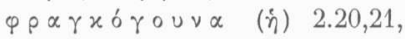

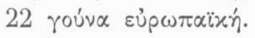

$\chi \alpha \sigma \dot{\varepsilon} \zeta \quad$ (o) $2.27,30,32 \cdot 4.19,25$, $29,35,37,39 \cdot 5.22$ ( $\chi \propto \sigma \alpha \tilde{\iota}, \chi \alpha \sigma \varepsilon)$

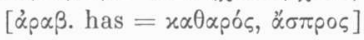

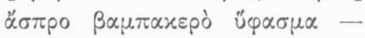

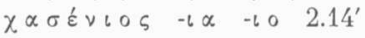

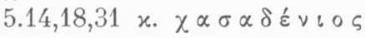

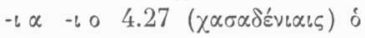

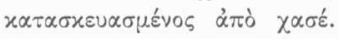

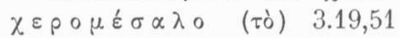
$\left(\chi \varepsilon \rho \circ \mu \varepsilon^{\prime} \sigma \alpha \lambda \alpha\right)\left[\chi^{\varepsilon} \rho \iota+\mu \varepsilon \sigma \alpha^{\prime} \lambda_{l}\right]$ $\pi \varepsilon \tau \sigma \dot{\varepsilon} \tau \alpha$.

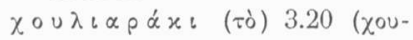

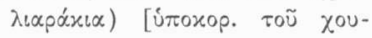

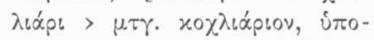

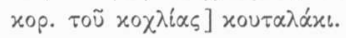




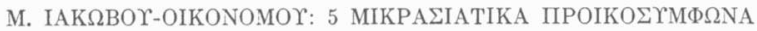

\section{TOП $\Omega$ NYMIA ${ }^{19}$}

'A $\gamma \dot{i} \alpha \Pi \alpha \rho \alpha \sigma x \varepsilon \cup \dot{\eta} 4.47,48$.

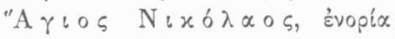
т०บั 3.8 .

'A $\pi \circ \sigma x \alpha \mu \nu i \varepsilon \varsigma 5.25$.

'A $p \tau \alpha \dot{\alpha} \eta \quad 3.29,54$.

'A $\varphi \cup \sigma \iota \dot{\alpha} 5.41$.

$\mathrm{B} \rho \propto \chi \nu \dot{\eta} 3.38$.

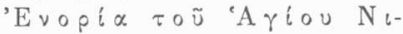
$x \circ \lambda \alpha \dot{\alpha} \circ 3.8$.

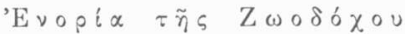
$\Pi \eta \gamma \tilde{\eta} s 3.35$.

'E $\pi \alpha \rho \chi\{\alpha \quad \mathrm{K} \cup \zeta i \varkappa \circ \cup \quad 4.7$.

$\mathrm{Z} \omega \circ \delta \delta \chi 0 s \quad \Pi \eta \gamma \dot{\eta}$, évopia ins 3.35 .
$\mathrm{K} \alpha \lambda \dot{v} \beta \propto 4.50$.

$\mathrm{K} \propto \mu i \nu \iota \propto 3.10$.

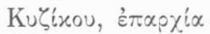

$4.7 \mid \mathrm{K} v-$ $\zeta i x \circ \cup 3.1$.

$M \propto \rho \mu \alpha \rho \tilde{\alpha} \varsigma \quad 2.45$.

$\mathrm{M} \eta \chi \alpha \nu \iota \dot{\omega} \nu \alpha \quad 4.6,59$.

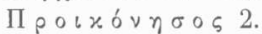

ПI $\rho$ ט $\sigma \alpha 3.45$.

$\sum x \dot{\alpha} \varphi \varepsilon \varsigma \quad 3.37$.

$\Sigma \mu \dot{v} \rho \nu \eta 1.47$.

$\sum u v 0 \iota x i \alpha \tau \tilde{\omega} v \quad T \alpha \xi \imath \alpha p-$ $\chi \tilde{\omega} \nu 2.33$.

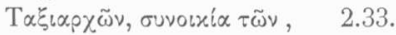
$\mathrm{T} \zeta \propto \varphi \alpha \rho \alpha 5.38$.

\section{KYPIA ONOMATA ${ }^{20}$}

"Aүros Kuל̧ixav, $\Sigma \varepsilon \beta(\alpha \sigma \mu \iota \omega$ $\tau \alpha \tau \circ \varsigma)$ ' $\mathrm{I} \varepsilon \rho \dot{\alpha} \varsigma \quad 3.1$,

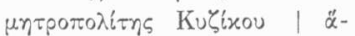

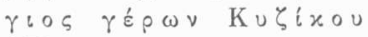
4.72.

"Artos II potxovñou

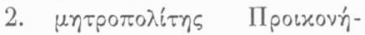
боบ.

'A $\theta \alpha \nu \alpha \sigma i \circ \cup$ Eu $\sigma \tau \dot{\alpha} \theta_{6} \circ \varsigma$ 1.49 .

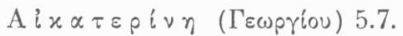

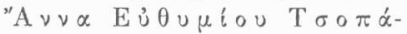

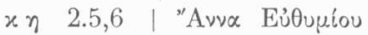

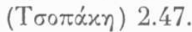

'A $\rho \chi \eta^{\prime \prime} \pi \alpha \pi \tilde{\alpha}$ 'E $\lambda \varepsilon \cup \theta \varepsilon \dot{\varepsilon} \rho$ ८

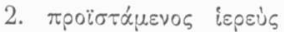

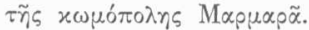

$\mathrm{B} \alpha \gamma \iota \alpha \gamma o ́ \mu \varepsilon v 0 \varsigma \quad \Theta$. 'A$\nu \propto \sigma \tau \dot{\alpha} \sigma$ :0૬ 4.64|| $\mathrm{B} \alpha-$

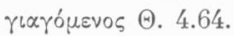

$\Gamma \alpha \nu \alpha \nu \tilde{\eta} \quad\left[\ddot{\eta} \Gamma \alpha \nu \alpha \dot{\alpha} \eta \ddot{\eta} \Gamma \alpha^{\prime} \nu \alpha \nu \eta\right]$ 'A

$\Gamma \varepsilon \omega \rho \gamma \iota 0 \varsigma$...... 4.

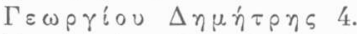

$\Gamma \varepsilon \omega p \gamma$ i०u $\mathrm{M}_{i} \chi \dot{\alpha} \lambda \eta \varsigma \mathrm{s}$ 4.66. $\Gamma \varepsilon \omega p \gamma i \circ v \quad \Pi \alpha v \alpha \gamma \iota \dot{\omega} \tau \eta \varsigma$

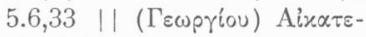
pivin 5.7.

$\Gamma \iota \propto \lambda \ldots . \quad \mathrm{X}^{\prime \prime}:$ I. $\mathrm{N} \iota x \circ \lambda \tilde{\eta} \varsigma$ $3.59 \| \Gamma\left\llcorner\alpha \lambda \ldots . . X^{\prime \prime}: I^{\prime} .3 .59\right.$.

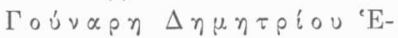
$\lambda \varepsilon \dot{\varepsilon} \eta \eta$ 3.3,5,6.

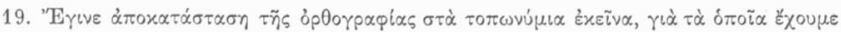

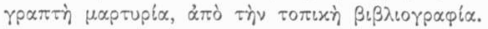

20. 'Е 


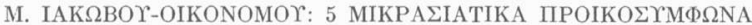

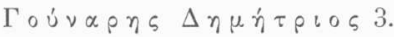

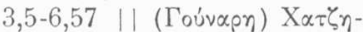
$\tau о \mu \nu \tilde{\eta} 3.33,57$.

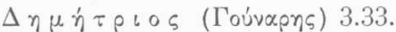

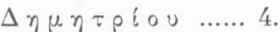

$\Delta \eta \mu \eta \tau \rho \hat{\imath} 0 \cup \quad \Gamma \varepsilon \dot{\omega} \rho \gamma \omega \circ \zeta$

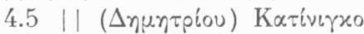

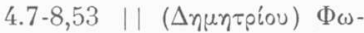
$\tau \varepsilon เ v \dot{n} 4.52$.

$\Delta \iota \alpha \mu \alpha \nu \tau i \delta \eta \varsigma$ Ф. 2.

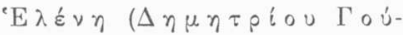
$v \propto \rho \eta) 3.24,34$.

$\mathrm{E} \lambda \varepsilon \cup \theta \varepsilon \rho\left\llcorner\sigma\right.$, , $\mathrm{A} \rho \chi \eta^{\prime \prime} \pi \alpha-$ $\pi \tilde{\alpha} \quad 2$.

'E $\lambda \varepsilon \cup \theta \varepsilon \rho \hat{\imath} \circ \cup \mathrm{K} \omega \nu \sigma \tau \alpha \nu-$ $\tau \tilde{\eta} \varsigma 5.7,|| \Sigma \tau \alpha u p \tilde{\eta}_{\varsigma} 5$.

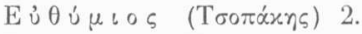
$\mathrm{Z} \alpha \chi \alpha \rho\{\alpha \zeta, \Pi \alpha \pi \tilde{\alpha} \quad 5$.

$\Theta \varepsilon \circ \delta \omega \rho \tilde{\eta} \varsigma 5.26,28$.

M $\cup \tau \dot{\alpha} \varphi \circ \gamma \lambda \circ \cup$ K. $\Theta \varepsilon \delta \delta \omega-$ p $\varsigma_{5} 3.32,3.35$.

$\Theta \omega \mu \tilde{\alpha} \mathrm{K} \omega \nu \sigma \tau \alpha \nu \tau \tilde{\iota} \nu \circ \varsigma 2.9$ $\mathrm{K} \omega \nu \sigma \tau \alpha \nu \tau \tilde{\eta} \varsigma 2.49$.

'I $\tau \zeta \varepsilon^{\prime} \sigma \varepsilon \lambda \eta \varsigma$ में 'I $\tau \zeta \varepsilon \varepsilon \pi \lambda \eta \zeta$ 'A. $\mathrm{N} \iota x \dot{0} \lambda \alpha \circ \varsigma 5.58$

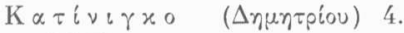
$7.8,53$.

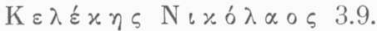

$\mathrm{K} \varepsilon \lambda \zeta \alpha \varphi \dot{u} \rho \eta$ 'A $\pi 0 \sigma \tau \dot{\sigma} \lambda \eta s$ 3.36 .

$\mathrm{K} \varepsilon \mu \pi \alpha \nu \iota \tilde{\alpha} \varsigma \Gamma \iota \dot{\alpha} \gamma x \circ \varsigma 3.39-$ 40.

$\mathrm{K} \circ \dot{u} \tau p \propto \varsigma \Gamma \varepsilon \dot{\omega} \rho \gamma \leftarrow \circ \varsigma 3.13$.

$\mathrm{K} \circ \dot{u} \tau p \varepsilon \nu \alpha$ 'E $\mathrm{E} \varepsilon \nu \mathrm{u} \dot{\omega} 3.9$.

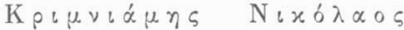
3.36 .

$\mathrm{K} \cup \rho\left\llcorner\dot{\alpha} \times \circ 5, \Pi \alpha^{\prime \prime} 2\right.$.

$\mathrm{K} v \sigma \tau \alpha \nu \tau \tilde{\imath} \nu \circ \varsigma \quad(\mathrm{M} 0 u \tau \dot{\alpha} \varphi 0$ $\gamma \lambda \circ \cup \tilde{\eta} \mathrm{M} \cup \tau \dot{\alpha} \varphi \circ \gamma \lambda \circ \cup 5)$ $3.3,5$.
$\Lambda \varepsilon \circ \nu \tau \tilde{\eta} \varsigma \Theta \varepsilon \circ \delta \omega \rho \tilde{\eta} \varsigma 5$.

$\Lambda \varepsilon \sigma i \rho \circ \gamma \lambda .0 \cup \varsigma \quad K \omega \nu \sigma \tau \alpha \nu-$ $\tau \tilde{\imath} \nu \circ \varsigma 3.3 \mathrm{u}$.

$\mathrm{M} \alpha \nu \alpha \sigma \alpha \dot{\alpha} x \varsigma 5.26-27$.

M $\sigma \eta p \lambda \sigma \gamma \lambda \circ \cup$ A. $\Theta$. 4.68.

M $\iota \alpha \dot{\eta} \lambda \Delta \eta \mu \dot{\eta} \tau \rho \iota \circ \varsigma 1.50$.

Mov $\tau \dot{\alpha} \varphi \circ \gamma \lambda \circ \cup \mathrm{K} \omega \nu \sigma \tau \alpha \nu$ $\tau \tilde{\imath}$ ○ $53.30, \mid 3.32$.

Mou $\alpha \dot{\alpha} \varphi \gamma \lambda \circ u \quad \Sigma 0 \varphi\{\tau \zeta \alpha$

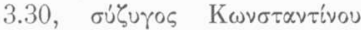

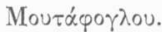

Mov $\tau \dot{\alpha} \varphi \circ \gamma \lambda \circ \cup \mathrm{K}$. $\Theta \varepsilon \sigma \delta \omega \rho \circ \varsigma$ 3.32 .

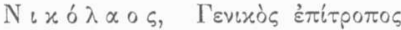

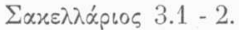

$\mathrm{N} \iota x \circ \lambda \tilde{\eta}_{\varsigma} \mathrm{X}^{\prime \prime}: \mathrm{I}^{\prime} . \Gamma\llcorner\alpha \lambda \ldots . .3 .59$

$\mathrm{N} \tau \circ$ ย $\tau$ ช $\quad(\mathrm{T} \dot{\alpha} \sigma \tau \alpha) 1.8$.

$\Pi \alpha \pi \tilde{\alpha} \quad \mathrm{Z} \alpha \chi \alpha \rho \hat{\imath} \alpha \varsigma \quad 5$. iepsis

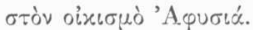

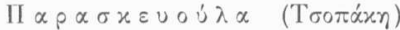
$2.7,35$.

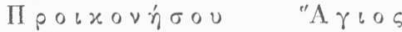
2.

$\Pi \rho \omega \tau 0 \pi \alpha \pi \tilde{\alpha} \quad \Gamma \varepsilon \dot{\omega} \rho \gamma: 0 \zeta$ 3.31 .

$\Pi \rho \omega \tau 0 \pi \dot{\alpha} \tau \sigma \eta \zeta$ 'I $\omega \dot{\alpha} \nu \vee \eta \zeta$ $1.9-10,50$.

$\Sigma \alpha \beta \beta \alpha \varsigma \quad \Gamma \varepsilon \omega .1 .48$.

$\Sigma \alpha x \varepsilon \lambda \lambda \alpha \rho \measuredangle 0 \varsigma \mathrm{N} \iota x b \lambda \alpha \circ \zeta$, $\Gamma \varepsilon \nu t x \dot{\partial} \zeta \quad$ ह่ $\pi i \tau \rho \circ \pi \circ \varsigma$, $3.1-2$.

$\Sigma \alpha \pi 0 u v \tau \zeta \tilde{\eta}$ 'A. 'A $\theta \alpha \nu \dot{\alpha}$ $\sigma \iota 0<4.69$

$\Sigma x \dot{\alpha} \rho \lambda \alpha \tau 0 u{ }^{\prime} \mathrm{E} \lambda \varepsilon \dot{\varepsilon} \vee \eta$ 4.6.

$\Sigma \tau \alpha \mu \alpha \tau \iota \alpha \delta \eta \varsigma$ A. 5.

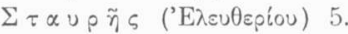

$\mathrm{T} \dot{\alpha} \sigma \tau \alpha \varsigma \quad \Gamma \varepsilon \dot{\omega} \rho \gamma \iota 05 \quad 1.7,4$

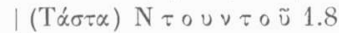

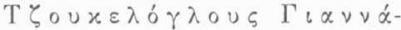
$x \eta 53.10-11$. 


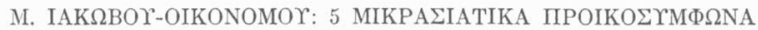

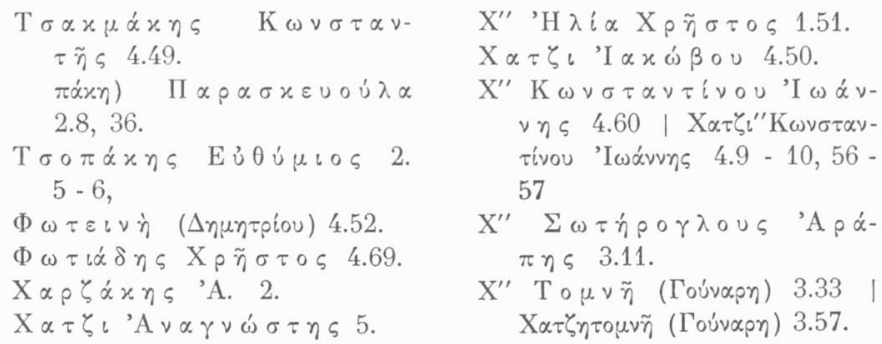

